\title{
Evolution of PHAS loci in the young spike of Allohexaploid wheat
}

\author{
Rongzhi Zhang ${ }^{1,2,3^{*}}{ }^{+}$, Siyuan Huang ${ }^{4 \dagger}$, Shiming $\mathrm{Li}^{4 \dagger}$, Guoqi Song ${ }^{1,2,3}$, Yulian Li ${ }^{1,2,3}$, Wei Li $\mathrm{Li}^{1,2,3}$, Jihu Li ${ }^{1,2,3}$, Jie Gao ${ }^{1,2,3}$, \\ Tiantian Gu, ${ }^{1,2,3}$, Dandan Li $i^{1,2,3}$, Shujuan Zhang ${ }^{1,2,3^{*}}$ and Genying $\mathrm{Li}^{1,2,3^{*}}$
}

\begin{abstract}
Background: PhasiRNAs (phased secondary siRNAs) play important regulatory roles in the development processes and biotic or abiotic stresses in plants. Some of phasiRNAs involve in the reproductive development in grasses, which include two categories, 21-nt (nucleotide) and 24-nt phasiRNAs. They are triggered by miR2118 and miR2275 respectively, in premeiotic and meiotic anthers of rice, maize and other grass species. Wheat (Triticum aestivum) with three closely related subgenomes (subA, subB and subD), is a model of allopolyploid in plants. Knowledge about the role of phasiRNAs in the inflorescence development of wheat is absent until now, and the evolution of PHAS loci in polyploid plants is also unavailable.
\end{abstract}

Results: Using 261 small RNA expression datasets from various tissues, a batch of PHAS (phasiRNA precursors) loci were identified in the young spike of wheat, most of which were regulated by miR2118 and miR2275 in their target site regions. Dissection of PHAS and their trigger miRNAs among the diploid (AA and DD), tetraploid (AABB) and hexaploid (AABBDD) genomes of Triticum indicated that distribution of PHAS loci were dominant randomly in local chromosomes, while miR2118 was dominant only in the subB genome. The diversity of PHAS loci in the three subgenomes of wheat and their progenitor genomes (AA, DD and AABB) suggested that they originated or diverged at least before the occurrence of the tetraploid AABB genome. The positive correlation between the PHAS loci or the trigger miRNAs and the ploidy of genome indicated the expansion of genome was the major drive force for the increase of PHAS loci and their trigger miRNAs in Triticum. In addition, the expression profiles of the PHAS transcripts suggested they responded to abiotic stresses such as cold stress in wheat.

Conclusions: Altogether, non-coding phasiRNAs are conserved transcriptional regulators that display quick plasticity in Triticum genome. They may be involved in reproductive development and abiotic stress in wheat. It could be referred to molecular research on male reproductive development in Triticum.

Keywords: PhasiRNAs, PHAS, MicroRNAs, Evolution, Young spike, Wheat

\section{Background}

There is a particular class of small RNAs generated in 21- or 24-nt (nucleotide) intervals with a 'head-to-tail' pattern from their precursor transcripts, which are called phased, secondary, small interfering RNAs (phasiRNAs) [1-3]. PhasiRNAs have been found both in animals and plants. The genes encoding PIWI-interacting RNAs

\footnotetext{
*Correspondence: zhangrongzhi1981@126.com; zsjhappy@163.com; Igy111@126.com

${ }^{\dagger}$ Rongzhi Zhang, Siyuan Huang and Shiming Li contributed equally to this work.

${ }^{1}$ Crop Research Institute, Shandong Academy of Agricultural Sciences, Jinan 250100, Shandong, China

Full list of author information is available at the end of the article
}

(piRNAs), which are a type of phasiRNAs, are required for producing mature sperm in animals [4]. In plants, phased siRNAs play a series of roles in abiotic and biotic stresses [5, 6], seed germination [7] and reproductive development [3, 8-10]. Trans-acting siRNAs (ta-siRNAs) are a special class of phasiRNAs generated from TAS precursors (noncoding or coding transcripts), which silence targets in trans. In addition, the regulation of disease resistance genes with the NB-LRR (nucleotidebinding sites and leucine-rich repeat) domains by miRNAs has mostly been characterized in the ETI (effectortriggered immunity) pathway of plants [11]. MiRNAs [12-14] can also trigger 21-nt phasiRNA generation from $N B-L R R$ transcripts, and most of them are species- 
specific. Some miRNA affects seed germination by generating phased siRNAs and modulating abscisic acid/gibberellin signaling in wheat [7].

In reproductive tissues, phasiRNAs are active in anther development of both eudicots and monocots. In grasses, there are two pathways that yield abundant phasiRNAs, which are associated with meiosis [3, 8]. MiR2118 triggers one class of 21-nt phasiRNAs in premeiotic anther development, while miR2275 triggers another class of 24-nt phasiRNAs [8]. In eudicots, 24-nt phasiRNAs are also present in the anther or pollen development triggered by either miR2275 or not [15]. This finding indicated the ancient origin of phasiRNAs and their regulatory mechanism. In general, $5^{\prime}$-capped and polyadenylated noncoding PHAS RNAs transcripted by RNA polymerase II could generate 21 - or 24-nt phasiRNAs, which mediates by miR2118 or miR2275, respectively. Then, the 3' mRNA fragments are converted into double-strand RNAs by RNA-dependent RNA polymerase 6 (RDR6), which are processed by DCL4 (dice like 4) or DCL5 (also named DCL3b) to yield 21- or 24-nt phasiRNAs [16]. Mutations in DCL4, DCL5 and RDR6 in rice affect the generation of 21- or 24-nt phasiRNAs [16, 17]. These phasiRNAs are subsequently loaded into AGOs to function their regulatory roles. In rice, MEL1 (also named OsAGO5c) preferentially binds to 21-nt phasiRNAs [18]. ZmAGO18b is enriched in the tapetum and germinal cells, and its expression pattern is similar to that of 24-nt phasiRNAs [8].

PhasiRNAs have been identified in a number of flowering plants in eudicots and monocots. In rice inflorescence, 828 and 35 of 21- and 24-PHAS were identified by Johnson et al [3], which could produce 21- and 24-nt phasiRNAs, respectively. In addition, 1136 and 1540 of 21-PHAS were identified in 93-11 and Nipponbare, respectively [9]. 70 and 34 of 24-PHAS were identified in 93-11 and Nipponbare by Song et al [16], respectively. In maize, 463 and 176 of 21-PHAS and 24-PHAS loci were identified [8]. In the flower of litchi, 178 of 21PHAS loci were identified [15]. These generation and regulation mechanisms are very conserved in grasses.

Numerous phasiRNAs are involved in the anther development process, which indicates that they may play a key role in normal anther development [8]. Until now, few phasiRNAs involved in developing inflorescence have been characterized in Triticum. Only miR9863 and miR9678 were characterized to mediate the generation of phasiRNAs in biotic stress [6] and seed germination [7], respectively. PhasiRNAs have been increasingly recognized as an important class of regulatory RNAs in several plant species. However, much remained unknown in the wheat genome regarding sequence information and expression levels of phasiRNAs, and the evolutionary path of phasiRNAs among their progenitors and the modern hexaploid wheat is still unclear. The large and complex genomes, including wheat and their progenitors (AA, DD and AABB genomes), are all available at the present. Furthermore, the number of small RNA datasets from high-throughput sequencing deposited in public databases, is increasing. And these datasets contain various developmental and stress samples of wheat. These efforts make it possible to systematically identify the phasiRNAs in wheat.

Here, using small RNA datasets in wheat, we investigated the PHAS loci in various tissues, such as leaves, roots, flag leaves, young spikes, and grain. The evolution of these PHAS loci in Triticum species indicated that their independently dynamic evolution was accompanied by their regulators miR2118 and miR2275. This study will be referred for the research of anther development in wheat.

\section{Results}

\section{Identification of 21- and 24-PHAS in wheat}

To identify the PHAS loci in wheat, we downloaded 261 small RNA datasets from the GEO database (Table 1), which included 12 seedling samples, 128 leaf samples, 12 root samples, one stem sample, one shoot sample, 29 young spike samples, two anther samples, one embryo sample, 17 spikelet samples, 12 rachis samples, 19 grain samples, 20 seed samples, 6 callus samples, and one mixed tissue sample. By comparing these small RNAs to the wheat genome using the Shortstack package [19] following the flowchart as shown in Supplementary Fig. 1, a batch of PHAS (phasiRNA precursors) loci were identified with phased scores greater than $15,20,25$ or 30

Table 1 The tissues of small RNA datasets used to identify the PHAS loci in wheat

\begin{tabular}{ll}
\hline Tissues & Number of samples \\
\hline Seedling & 12 \\
Leaf & 128 \\
Root & 12 \\
Stem & 1 \\
Shoot & 1 \\
Young Spike & 29 \\
Anther & 2 \\
Embryo & 1 \\
Spikelet & 17 \\
Rachis & 12 \\
Grain & 19 \\
Seed & 20 \\
Callus & 6 \\
Mixed tissues & 1 \\
Total & 261 \\
\hline
\end{tabular}


(Supplementary Table 1). In the reproductive tissues such as young spikes and anthers (Fig. 1a-b), abundant 21- and 24-PHAS were identified. However, In the leaf, stem, root, spikelet, seed, callus, etc., few PHAS were identified (Supplementary Table 1 \&\& Supplementary Fig. 2). The abundance of phasiRNAs was not correlated with the total reads of the small RNA samples, but it was highly related to the types of tissues (Supplementary Fig. 2).

In the downloaded small RNA samples of wheat, there were a group of datasets, which included the important development stage of young spike [20], i.e., DR stage (doubleridge stage) at the initiation of spike formation and spikelet development; FM stage, the stage of appearance of the floret meristems (FMs) glume primordia, and lemma primordia; AM stage (anther primordia stage), stamen and pistil primordia emerged from FMs with visible anther primordia for some florets; TS stage (tetrads stage), young florets began to differentiate with immature anthers and unelongated pistils, and the pollen mother cells completed meiosis to form the tetrads at this stage. In the DR and FM stages of young spike tissues, there were few PHAS loci, while in the AM and TS stages, there were abundant 21- and 24PHAS. For example, $\sim 3600$ and $\sim 4000$ of the 21-PHAS loci with phased scores greater than 30, and 1200 and 1000 of the 24-PHAS loci with phased scores greater than 30 were identified in AM (SRR3690677 and SRR3690678) and TS (SRR3690679 and SRR3690680) samples, respectively

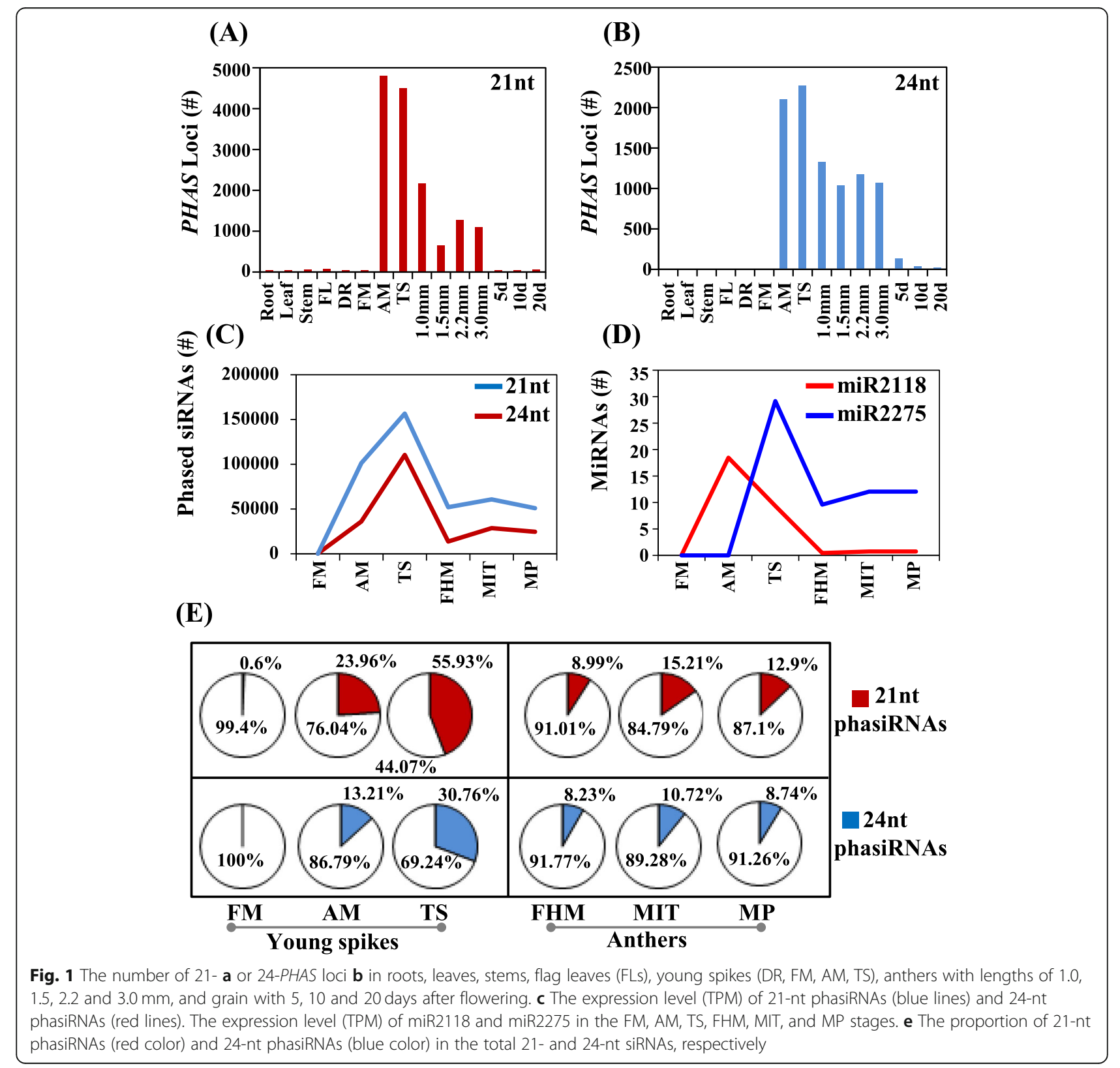


(Supplementary Table 1). The number of 21-PHAS loci in anthers with lengths of $1.0 \mathrm{~mm}$ were more than in those with lengths of 1.5, 2.2 and $3.0 \mathrm{~mm}$, while for the 24-PHAS, the number of $P H A S$ loci were very similar among the different length of anther (Fig. 1a-b). According to morphological development and stage determination of young spike and anther in wheat [21], six small RNA datasets were selected for further study that represented the early and later anther development stages. The early anther development stage included the floret meristem (FM, SRR5460941 and SRR5460949), anther primordia (AM, SRR5460967 and SRR5460972) and tetrad stages (TS, SRR5461176 and SRR5461177), and the later anther development stage included free haploid microspores (FHM, SRR449365 with $1.5 \mathrm{~mm}$ anther), mitosis (MIT, SRR449366 with $2.2 \mathrm{~mm}$ anther), and mature pollen (MP, SRR449367 with $3.0 \mathrm{~mm}$ anther). In the next section, these PHAS with score more than 30 were selected to do the further analysis.

During wheat inflorescence development, the expression level of 21-nt phasiRNAs at one particular PHAS locus may vary at different stages. In the FM stage, there were few 21-nt phasiRNAs (300 TPM (transcripts per million)) with $0.6 \%$ out of the total 21-nt small RNAs, while in AM, a sharp increase (36,054 TPM) was observed, comprising $23.96 \%$ out of the total. In the TS stage, the expression level continued to increase with 11, 410 TPM of 21-nt phasiRNAs (44.07\% out of the totals) (Fig. 1c \& e). For the 24-nt phasiRNAs, the tendency of the proportion was similar to that of the $21-n t$ phasiRNAs in the three stages. The proportions of 24-nt phasiRNAs out of the total 24-nt siRNAs were $0,13.21 \%$ (101,277 TPM), and $30.76 \%$ (156,595 TPM) for the FM, AM, and TS stages, respectively (Fig. 1c \& e). This indicated that the 21- or 24-nt phasiRNAs and 21- or 24PHAS loci were present in the AM stage and peaked in the TS stage. For the later anther development stage, the 21- and 24-nt phasiRNAs occurred in the FHM stage with proportions of $8.99 \%(13,701 \mathrm{TPM})$ and $8.23 \%$ (52, $030 \mathrm{TPM})$, peaked in the MIT stage with proportions of $15.21 \%(28,633 \mathrm{TPM})$ and $10.72 \%(60,758 \mathrm{TPM})$, and then decreased with proportions of $12.9 \%$ (24,562 TPM) and $8.74 \%$ (50,908 TPM), respectively (Fig. 1c \& e). The proportions of 21- and 24-nt phasiRNAs in the three later anther development stage, were much lower than that of the AM or TS stage.

The synthesis of phasiRNAs in monocot reproductive tissues requires both PHAS precursors and their initiated miRNAs, such as miR2118 and miR2275 [16, 17]. The expression level of miRNAs was concertedly related to the synthesis of the phasiRNAs and PHAS loci. For 21and 24-PHAS, the PHAS loci peaked both in AM and TS, and then rapidly decreased in the later anther development stage (Fig. 1a-b). Both 21- and 24-nt phasiRNAs were expressed in AM, peaked only in TS and rapidly descended in the later anther development stage (Fig. 1c $\& \mathrm{e})$. We then investigated the concert of the three elements including PHAS, phasiRNAs and their regulated miRNAs at the transcriptome level. The expression of miR2118 peaked in AM, which was similar to that of 21PHAS, but occurred before the burst of 21-nt phasiRNAs. Then, the expression of miR2118 decreased in TS and disappeared in the later anther development stage. The expression of miR2275 peaked in TS, which was before that of 24-nt phasiRNAs but the same as that of 24PHAS. Then, the expression of miR2275, 24-nt phasiRNAs and 24-PHAS rapidly decreased in the later anther development stage (Fig. 1a-e). The expression of miR2275 was higher than that of miR2118 in the later anther development stage, which may be associate with the higher expression of 24-nt phasiRNAs than 21-nt phasiRNAs in these stages (Fig. 1a-e). Together, the expression of the 21- and 24-nt phasiRNAs and their trigger miRNAs both burst in the early anther development stage.

\section{The distribution of PHAS loci in the wheat genome}

To investigate the genome distribution of PHAS in wheat, we used the Circos package to show the number of PHAS loci sliding the chromosome in window sizes of $500 \mathrm{~kb}$. Here, we selected these small RNA datasets with abundant phasiRNAs in AM, TS, FHM, MIT and MP for further study. In the five developmental stages, the distribution of genome elements such as repeat sequences, gene body and intergenic regions were very similar in both 21 - and 24-PHAS (Supplementary Fig. 3). Few PHAS were located in the gene body or repeat sequence regions in the genome. Only $1 \sim 2 \%$ of these PHAS loci were distributed in gene body regions, and $12 \sim 21 \%$ of them were distributed in repeat sequence regions. In contrast, most of them (78 87\%) were located in the intergenic regions. This result indicates that most PHAS loci may have independent transcript units that are not juxtaposed with the repeat sequences or coding genes.

According to the genome locations, we respectively merged all of the 21- and 24-PHAS in these samples derived from five inflorescence development stages. In total, there were 4850 and 3600 of unique 21- and 24PHAS in these samples, respectively (Supplementary Fig. 4A-B), most of which were common. In total, $94.93 \%$ (2042 out of 2151), 53.14\% (2385 out of 4488), $98.61 \%$ (637 out of 646$), 94.85 \%$ (1263 out 1198) and 95.91\% (1056 out of 1101) of 21-PHAS (Supplementary Fig. 4A), and $49.87 \%$ (993 out of 1991), 69.09\% (1571 out of 2274), $98.26 \%$ (1019 out of 1037), 98.38\% (1157 out of 1176 ) and $98.79 \%$ (1057 out of 1070) of 24-PHAS (Supplementary Fig. 4B), were overlapped each other in at least two samples in AM, TS, FHM, MIT, and MP, respectively. The low number of common 21-PHAS in TS 
and 24-PHAS in AM indicated that there may be more tissue-specific PHAS loci in these two development stages, which may be associated with the transition of the development stage from floret meristem to meiosis.

These merged unique PHAS were plotted on the chromosome rainbows with black lines (Fig. 2). Most of the PHAS loci were located at the end of the chromosomes, i.e. telomere regions. In most regions, the peaks of the 21-PHAS (red lines in Fig. 2) were higher than those of the 24-PHAS (blue lines in Fig. 2) in the representative tissues. Most of the peaks in both 21- and 24-PHAS were similar among the subA, subB and subD genomes in each sample. However, some peaks of 21and 24-PHAS in local chromosomes were preferred among the three subgenomes in each sample (red and blue arrows in Fig. 2 and Fig. 3).

\section{The genome plasticity of PHAS in Allohexaploid wheat} Polyploidization is followed by genome partitioning or fractionation processes, i.e. a genome-wide diploidization, in which one or the other gene duplicate is lost. During this process, differently functional protein-coding genes have been shown to behave differently. Transcription factors or regulators are often retained as duplicated copies following whole genome duplications (WGDs), whereas others are progressively deleted back to a single copy (singleton) state [22-24]. For allohexaploid wheat (AABBDD, Triticum aestivum, $2 \mathrm{n}=6 \mathrm{x}=42$ ), high sequence similarity and structural conservation are retained with limited gene loss after polyploidization [25]. However, at the transcript level, cell type- and stage-dependent genome dominance was observed in the local chromosome regions [26]. Studies of the roles of PHAS loci as a category of noncoding genes in the partitioning process of allohexaploid wheat have still not been performed. To investigate the location of PHAS in the subA, subB and subD of allohexaploid wheat, we performed a blast search against all genomes to identify the relationship of PHAS among the three subgenomes. With $80 \%$ identity and $80 \%$ matched sequence length, only $2.27 \% \sim 6.40 \%$ of PHAS in

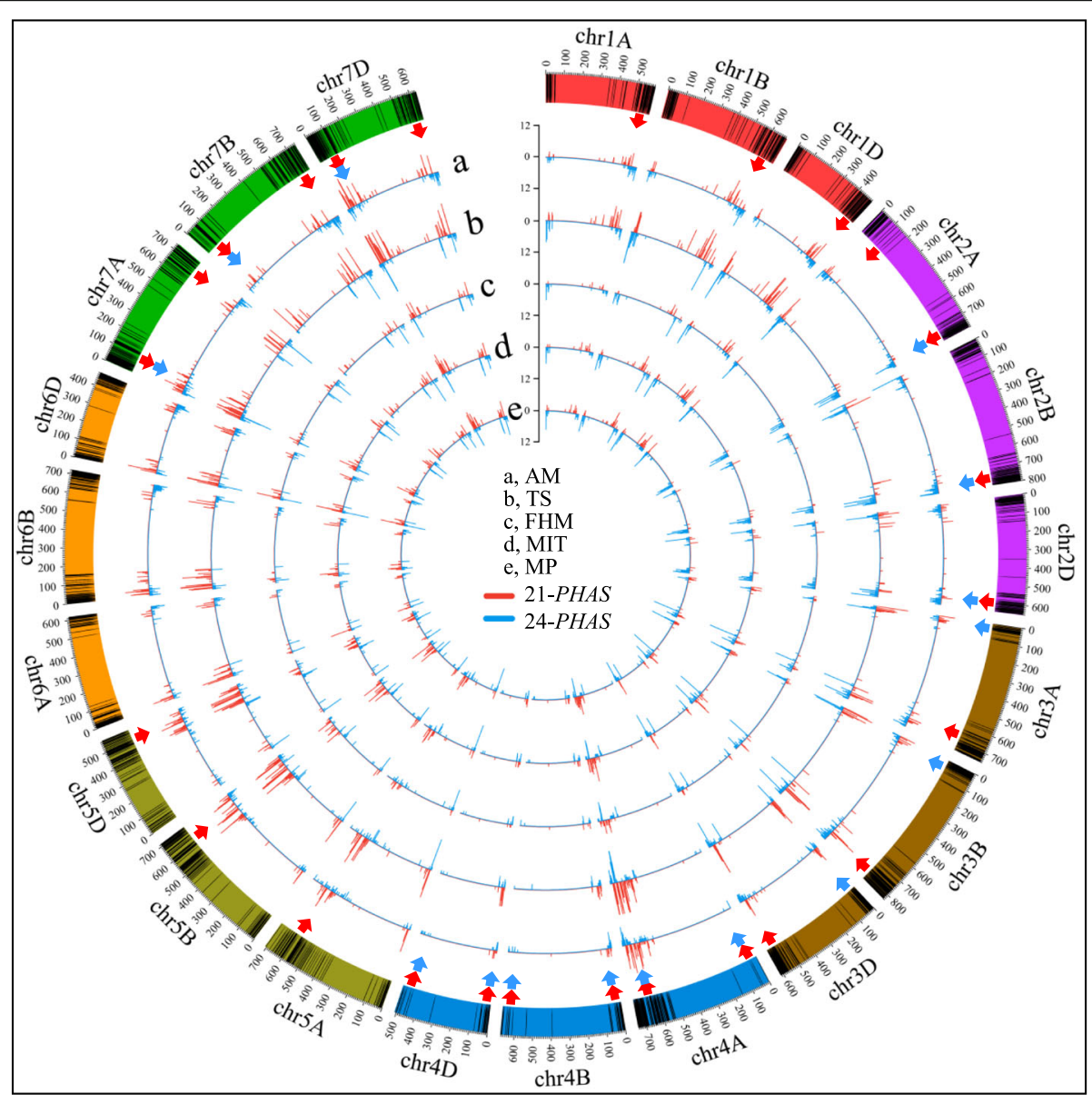

Fig. 2 Density distribution of 21- (red lines) and 24-PHAS loci (blue lines) in the young spike samples. The black lines in the chromosome represent the PHAS loci. The peaks in circles a-e indicate the number of PHAS loci in each $500 \mathrm{~kb}$ region across each chromosome in AM, TS, FHM, MIT and MP, respectively. The red and blue arrows represent the biased distribution of 21- and 24-PHAS among the three subgenomes, respectively 


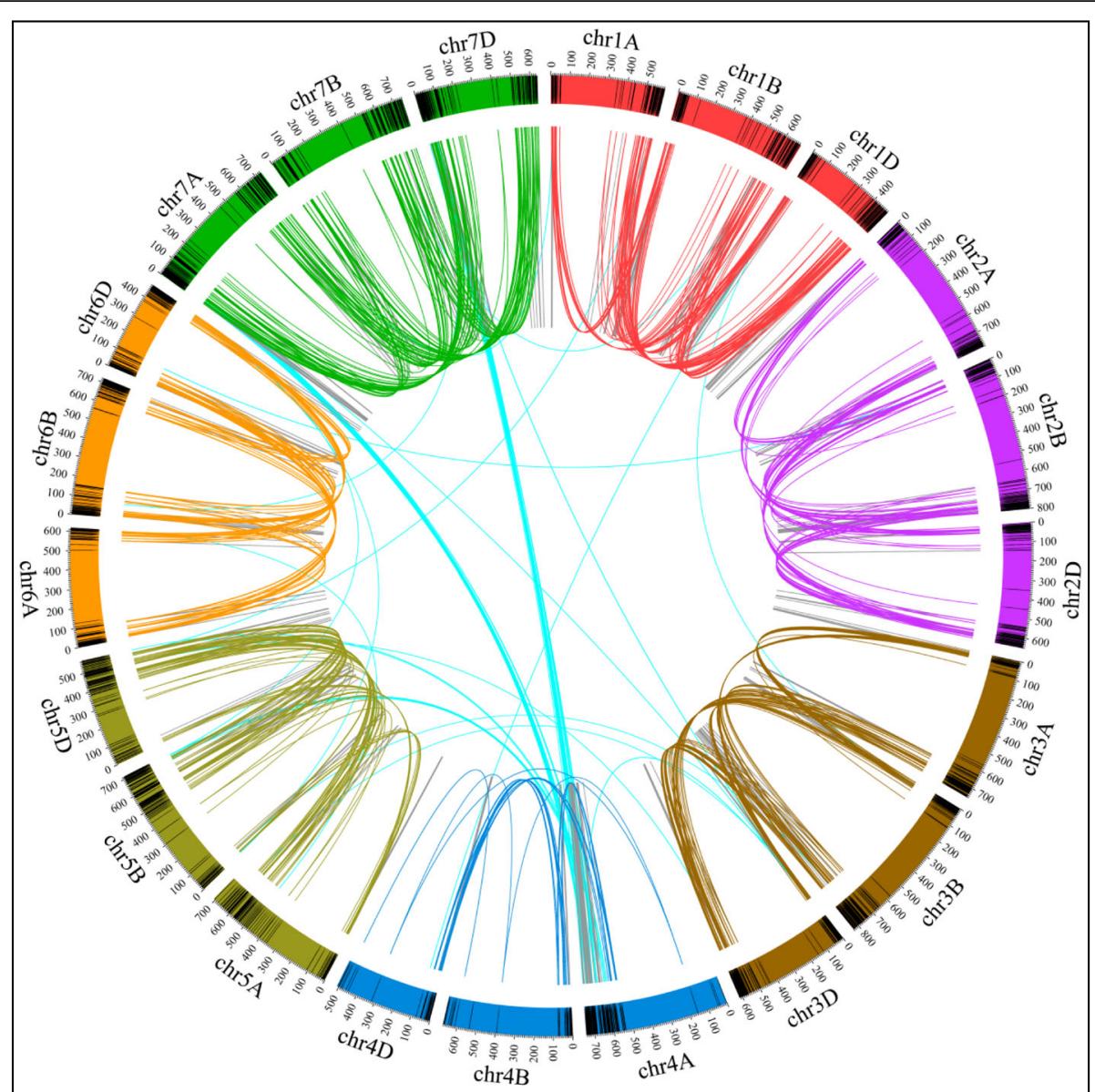

Fig. 3 The duplication relationship among the three homoeologous chromosomes. The homoeologous PHAS loci are linked by lines with the same color. The black bar represents the PHAS loci in each chromosome

the five samples retained the triplet copies in the three subgenomes, $11.11 \% \sim 17.51 \%$ of PHAS retained the duplet copies in any two subgenomes, and $76.09 \% \sim 86.22 \%$ of PHAS retained only singleton in any one subgenome (Supplementary Fig. 5). The homoeologous relationship is shown in Fig. 3 with the same color link lines in the homoeologous chromosomes. There were also some translocated homoeologs (the cyan links in Fig. 3). For example, some PHAS in chr4A were homologous to those in chr5B/D and chr7A/D. These data showed that only partial PHAS retained the triple or duplet homoeologs, and most PHAS only possessed the singleton copy.

To investigate the subgenome distribution of PHAS loci in allohexaploid wheat, the total number of PHAS loci in each sub-chromosome was calculated, and there were no biased in each subA, subB and subD chromosomes. However, for the local chromosomes, there were some bias distribution in the local subA, subB and subD genomes. For 21-PHAS loci, in the bottom chromosomes of chr1, chr2, chr3 and chr4, and in the top and bottom chromosomes of chr4 and chr7, the PHAS loci were biased located in the chromosomes, but the tendency of preference was different, in either the top or bottom of one chromosome (Fig. 2 (the red arrow regions) and Fig. 4a). In chr1-b (b, bottom of the chromosome), significantly less 21-PHAS were located in the subA genome than in the subB and subD genomes (Fisher's exact test, $P$-value $<0.05)$. In chr2-b, the number of 21-PHAS was significantly less in subB than in subA and subD (P-value $<0.05)$. In chr3-b, subB became the dominant genome with significantly more 21-PHAS than the subA and subD genomes $(P$-value $<0.05)$. In chr4-t (top of the chromosome), the number of 21-PHAS was less in subA than in subB and subD $(P$-value $<1.0 \mathrm{e}-5)$, while in chr4-b, the subA genome possessed numerous 21-PHAS, significantly more than the other subgenomes $(P$-value $<2.2 \mathrm{e}-16)$. In chr7-t, subB possessed less of the phased loci, next to the subA genome, and the subD possessed much more 21-PHAS than subA and subB $(P$-value $<0.001)$.

For 24-PHAS, only chr3-t, chr4-t/b and chr7-t in all five samples exhibited a biased distribution (Fig. 2 (blue 

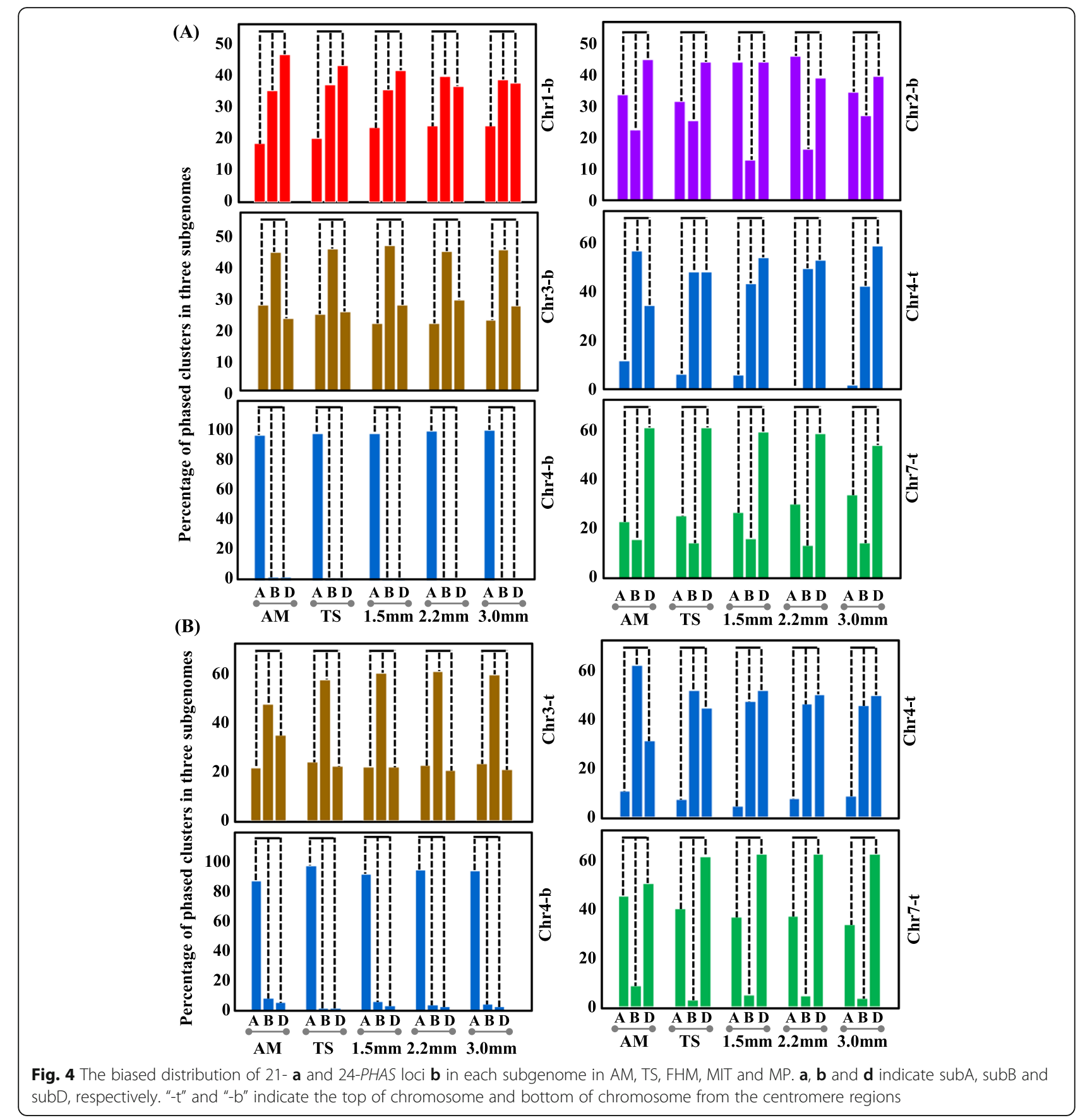

arrow regions) and Fig. 4b). In chr4-t/b, the preference of 24-PHAS in chromosomes was very similar to that of 21-PHAS. In chr4-t, less PHAS loci were located in subA than in subB and subD (P-value $<1.0 \mathrm{e}-6)$, while in chr4$\mathrm{b}$, more $P H A S$ loci were located in subA than in subB and subD (P-value $<2.2 \mathrm{e}-6)$. In chr3-t, more 24-PHAS were distributed in the subB genome than in the subA and subD genomes $(P$-value $<0.05)$. However, in chr7-t, far fewer 24-PHAS were located in the subB genome than in the subA and subD genomes $(P$-value $<1.0 \mathrm{e}-6)$.
These data suggested that PHAS loci exhibited local chromosome preferences during the genome plasticity process.

\section{Homoeologous PHAS loci in the diploid, Tetraploid, and Hexaploid wheat}

The progenitors of allohexaploid wheat contain the diploid genomes $\mathrm{AA}, \mathrm{BB}$ and $\mathrm{DD}$, and the tetraploid genome AABB. The genomes of AA (Triticum urartu, $2 \mathrm{n}=2 \mathrm{x}=14$ ), DD (Aegilops tauschii, $2 \mathrm{n}=2 \mathrm{x}=14$ ), and 
AABB (Triticum turgidum, $2 \mathrm{n}=4 \mathrm{x}=28$ ) were sequenced, and their genome assembly was nearly perfect at the chromosome level. This made it possible to investigate the evolution of 21- and 24-PHAS in the different ploidy of Triticum species. Thus, we downloaded the genome sequences of $\mathrm{AA}, \mathrm{DD}$ and $\mathrm{AABB}$, and then mapped the 21- and 24-PHAS with scores of greater than 30, which were identified in these samples from the five developmental stages of inflorescence, to these genome sequences by the BLAST program with $80 \%$ identity and $80 \%$ matched sequence length. Approximately $22.91 \% \sim 25.98 \%$ of the $21-$ PHAS could be mapped to the AA genome, and $37.77 \% \sim 44.12 \%$ of the 21 -PHAS could be mapped to the DD genome, which was slightly higher than the 21-PHAS that were mapped to the AA genome. However, a greater proportion of PHAS $(60.83 \%$ $62.72 \%$ ) could be mapped to the AABB genome. For 24PHAS, the mapped proportions to $\mathrm{AA}, \mathrm{DD}$ and $\mathrm{AABB}$ were very similar to the mapped 21-PHAS. Approximately $22.52 \% \sim 26.13,29.18 \% \sim 37.42$, and $53.96 \%$ $58.05 \%$ of $24-P H A S$ could be aligned to the AA, DD and
AABB genomes, respectively (Supplementary Table 2). In the tetraploid AABB, the mapping rates of 21- and 24PHAS were much higher than the diploid species AA and $\mathrm{DD}$. The scatter diagram showed that the mapping rate was positively correlated with the ploidy times in 21-PHAS with $\mathrm{r}^{2}=0.8019$, as determined by Pearson correlation test $(P$-value $=6.41 \mathrm{e}-6$, Supplementary Fig. 6A $)$, and 24-PHAS with $\mathrm{r}^{2}=0.8578 \quad(P$-value $=6.41 \mathrm{e}-6, \quad$ Supplementary Fig. 6B). This indicated that the expansion of the whole genome led to an increase of the PHAS loci.

For the mapped 21- and 24-PHAS to the AA genome, 67.55 and $75.73 \%$ of them were located in the subA genome of wheat, respectively. For the mapped 21- and 24PHAS to DD genome, there were 72.78 and $77.53 \%$ of them located in subgenome D of wheat, respectively. For the mapped 21-PHAS to the AABB genome, 43.41 and $42.40 \%$ of them were from the subA and subB genome of hexaploid wheat, respectively, and for 24-PHAS, a similar proportion (i.e. 44.38 and $44.51 \%$ for subA and subB, respectively) was observed. The detailed genome distributions in the AABBDD genome for the mapped PHAS were

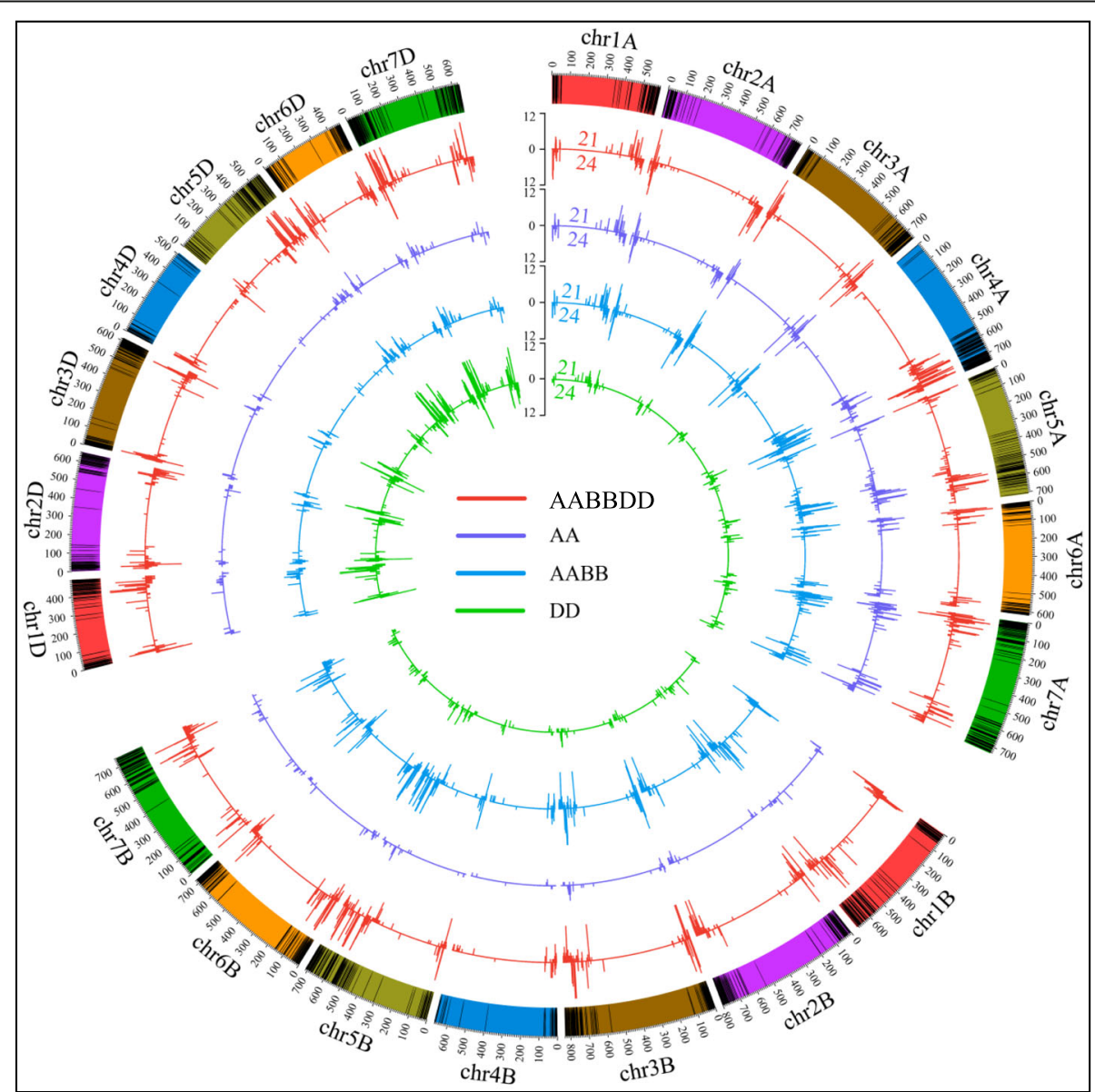

Fig. 5 The density of 21- (top lines) and 24- (bottom lines) PHAS loci that can be mapped to the AABBDD (red lines), AA (purple lines), AABB (blue lines) and DD (green lines) genomes. The black bar represents the PHAS loci in each chromosome 
clearly shown in each chromosome of Fig. 5. For the PHAS mapped to the AA genome, there were more peaks (purple lines in Fig. 5) from each chromosome of subA than from the other subgenomes. For the mapped PHAS to the DD genome, the peaks (green lines in Fig. 5) were mostly distributed in the subD genome. For the mapped PHAS to the AABB genome, each chromosome of both subA and subB had more peaks (blue lines in Fig. 5) than the subD genome. This finding indicated the orthologous relationship between each of subA and the AA genome, subB and $\mathrm{BB}$ genome, and subD and DD genome. The evolution independence of $P H A S$ sequences among the three diploid species, $\mathrm{AA}, \mathrm{BB}$ and $\mathrm{DD}$, may suggest that the PHAS sequences in Triticum may diverge before tetraploid synthesis and may diverge after occurrence of the $\mathrm{AA}, \mathrm{BB}$ and $\mathrm{DD}$ species.

\section{Genome distributions of MiR2118 and MiR2275 in Triticum}

The production of phasiRNAs was initiated by miR2118 and miR2275 via cleavage of their PHAS precursors. MiR2118 and miR2275 possessed many copies in grasses. There were 18 members of miR2118 and four members of miR2275 in the rice genome and seven members of miR2118 and four members of miR2275 in the maize genome, based on the miRBase database [27]. To detect these miRNAs in Triticum, we mapped the mature sequences of miR2118 and miR2275 from the miRBase database to the Triticum genomes with perfect matches and found that there were $25,30,88$, and 140 members of miR2118 in the DD, AA, AABB and AABBDD genomes, respectively, and 6, 5, 17 and 24 members of miR2275 in the four genomes, respectively. Most of these miRNAs were clustered on the chromosomes of the four species, as shown in Fig. 6a and Supplementary Fig. 7-8. The increase tendency of miR2118 and miR2275 was significantly correlated with ploidy, as determined by Pearson correlation test $(P$-value $=0.0015$ and $0.0084, \mathrm{r}^{2}=0.9971$ and 0.9833 , respectively; Fig. $6 \mathrm{~b}-$ c). For miR2118, there was also a biased distribution in the three subgenomes of wheat. However, unlike the 21PHAS, the tendency of miR2118 on the subgenome in each group of chromosomes was consistent (Fig. 6a). Except for chr2, subB was significantly dominant, with many more members of miR2118 than the subA and subD genomes in chr1 (Fisher exactly test, $P$-value $=$ $1.87 \mathrm{e}-5)$, chr4 $(P$-value $=3.90 \mathrm{e}-14)$ and $\operatorname{chr} 5(P$-value $=$ $3.65 \mathrm{e}-14)$. On chr2, there were also more members of miR2118 in the subB genome but not significantly with $P$-value $>0.05$. In the tetraploid AABB genome (Supplementary Fig. 7), miR2118 in subB was also dominant than the subA genome on chr1 $(P$-value $=2.06 \mathrm{e}-8)$, chr2 $(P$-value $=1.43 \mathrm{e}-2)$, chr $4(P$-value $=3.72 \mathrm{e}-10)$, and chr5 $(P$-value $=1.43 \mathrm{e}-2)$. The similar dominant subgenome distribution of miR2118 in the AABB and AABBDD genomes indicated that the dominance of subB may have occurred before the synthesized hexaploidy of wheat. In the AA and DD genomes, there were also fewer miR2118 in chr1, chr2, chr4 and chr5 than in subB of the AABB and AABBDD genomes (Supplementary Fig. $8 \mathrm{~A}-\mathrm{B})$, which indicated that the expansion of miR2118 in the subB genome may have occurred before the synthesized tetraploidy of the AABB genome. This indicated the dynamic expansion of the trigger miRNAs following genome expansion or polyploidization.

\section{PHAS loci as the targets of MiR2118 and MiR2275 in wheat}

The production of the PHAS loci could be initiated by miR2118 and miR2275, and then generate the 21- and 24nt phasiRNAs, respectively. To identify whether miR2118 and miR2275 also target these PHAS transcripts in wheat, using the Targetfinder program [28], we aligned the two miRNA families to the PHAS sequences with a score less than 4. Taking these samples of the five development stages as examples, $35.43 \%$ (767 out of 2159), $34.06 \%$ (220 out of 646 ), 35.31\% (446 out of 1263), 38.31\% (824 out of 2151), and $35.09 \%$ (1575 out of 4488) of 21-PHAS sequences were predicted to be targeted by miR2118 in AM, ST, FHM, MIT, and MP, respectively. For 24-PHAS, $43.36 \%$ (575 out of 1323), 50.43\% (523 out of 1037), $50.26 \%$ (591 out of 1176), $0.45 \%$ (9 out of 1991), and $48.86 \%$ (1111 out of 2274 ) were identified as the targets of miR2275, respectively. The alignment information between the miRNAs and PHAS loci were listed in Supplementary Table 3, 4, 5, 6, 7 .

To validate whether these PHAS transcripts can be indeed cleaved by miR2118 and miR2275, we downloaded the degradome sequences from the GEO datasets for the young spike tissue under cold stress by Song et al [29], which corresponds to the small RNA datasets of the AM stage of young spike (control samples, SRR3680677 and SRR3680678; and cold stress samples at $0{ }^{\circ} \mathrm{C}$ after $48 \mathrm{~h}$, SRR3680679 and SRR3690680). According to the abundance of reads along the whole transcripts, using the Cleaveland program [30] (P-value $<0.05$ and the category $<=2$ ), miR2118 and miR2275 were considered to be interacted between PHAS transcripts and miRNAs. The target plots of miR2118 and miR2275 characterized in the degradome datasets were shown in Fig. 7a-d. And at the cleavage sites, the generated phasiRNAs with orientation from the positive or negative strand were shown in the bottom panel of Fig. 7a-d. A total of $13.01 \%$ (520 out of 3996, SRR3680679) and 12.80\% (525 out of 3996 in SRR3680680) of 21-PHAS were confirmed to be cleaved by miR2118 for the cold stressed samples. In the control samples, slightly less 21-PHAS were validated as cleaved targets, i.e. 9.86\% (355 out of 3601 in 


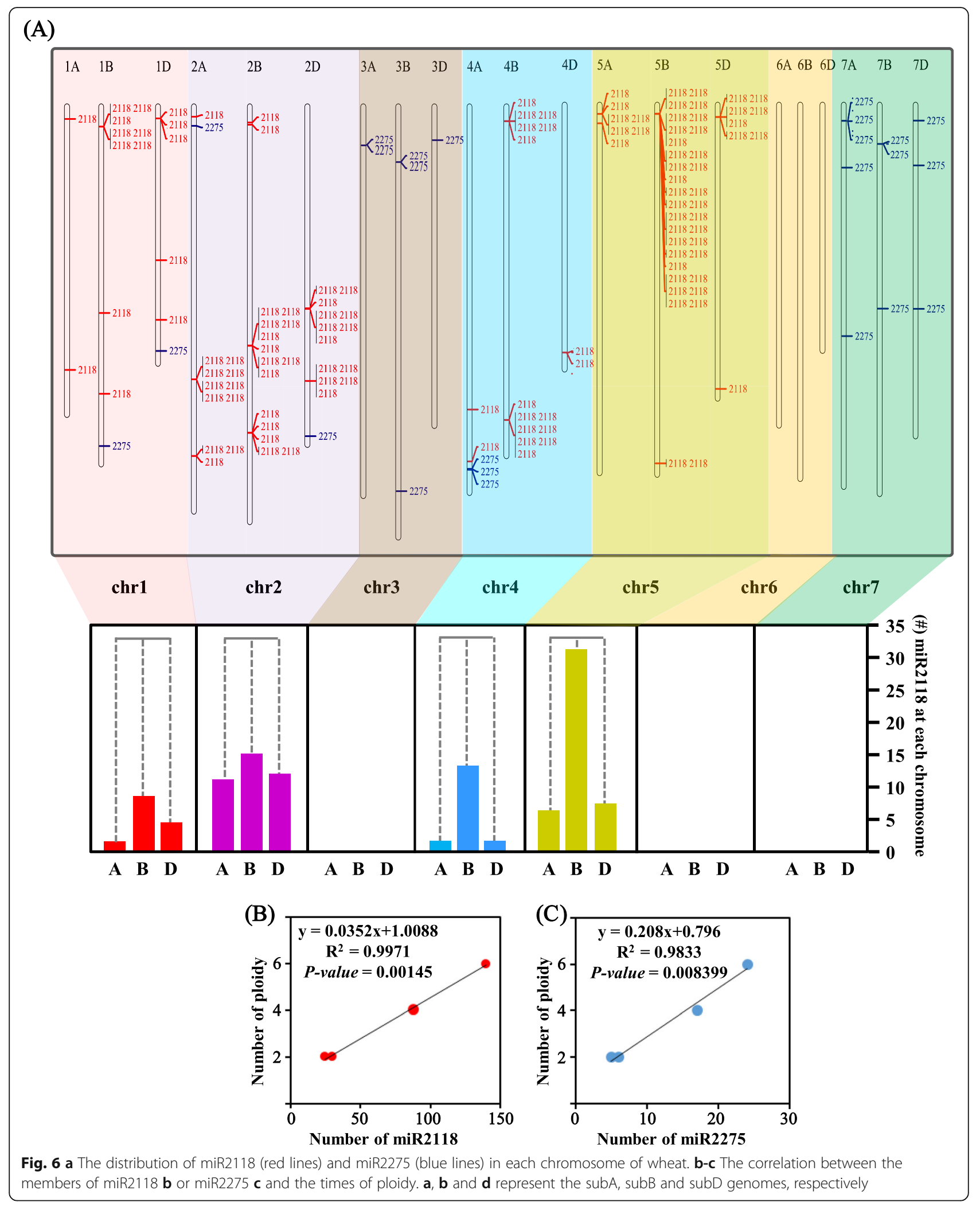


(A)
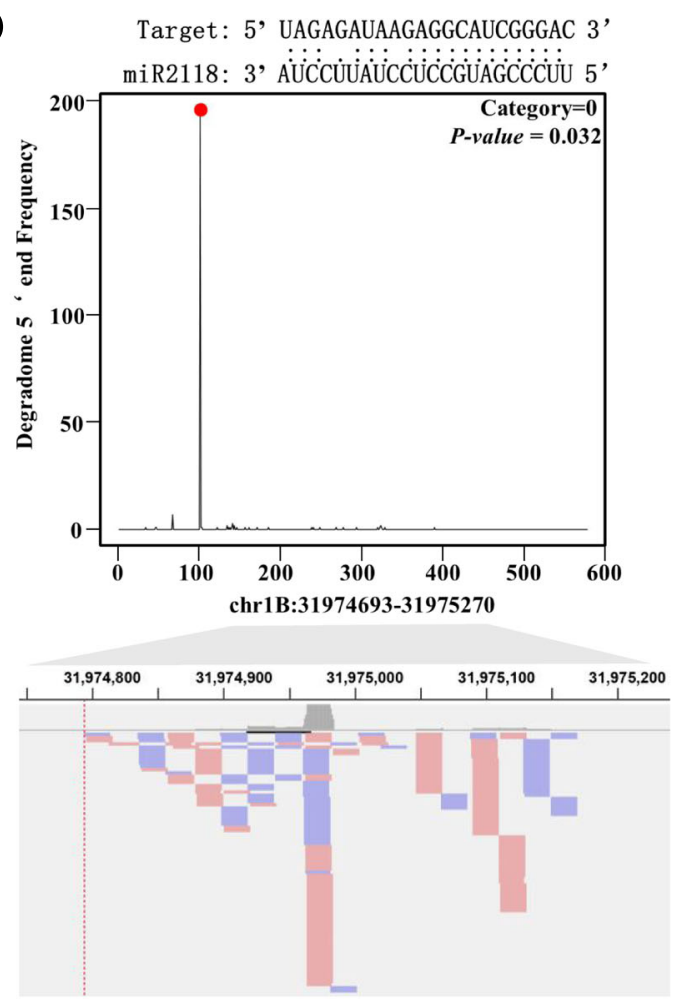

(C)

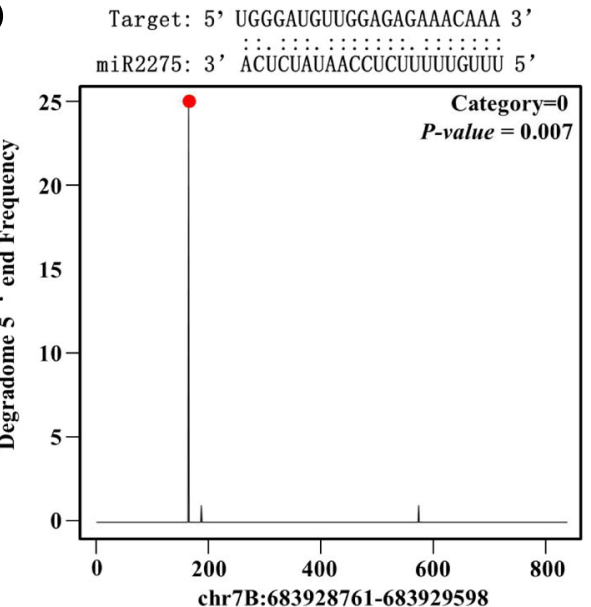

$683,928,900 \quad 683,929,000 \quad 683,929,100 \quad 683,929,200683,929,300 \quad 683,929,400 \quad 683,929,500$

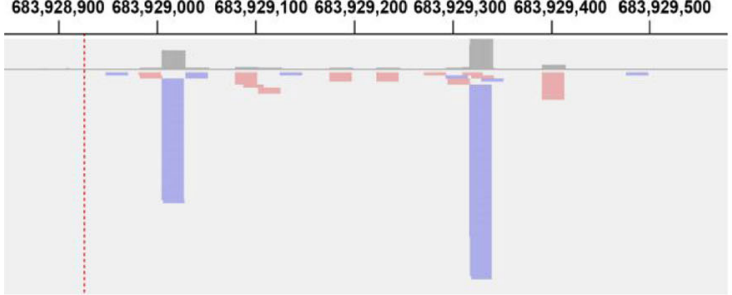

(B) Target: 5, UGGGAGUAGGAGGUAGCAGGAA 3,

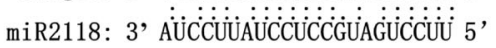

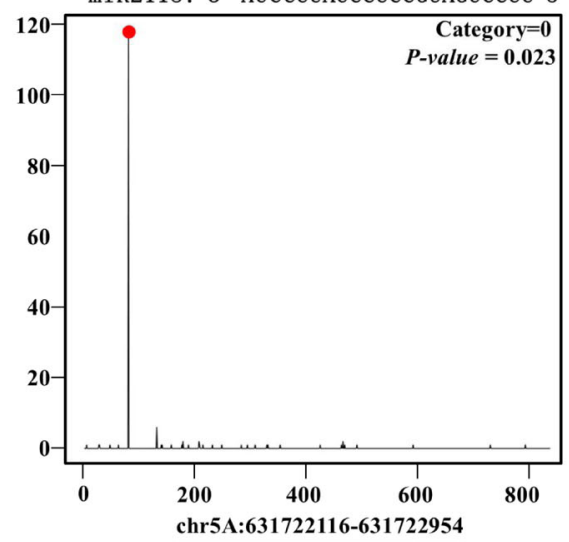

$631,722,200631,722,300631,722,400631,722,500631,722,600631,722,700631,722,800631,722,900$

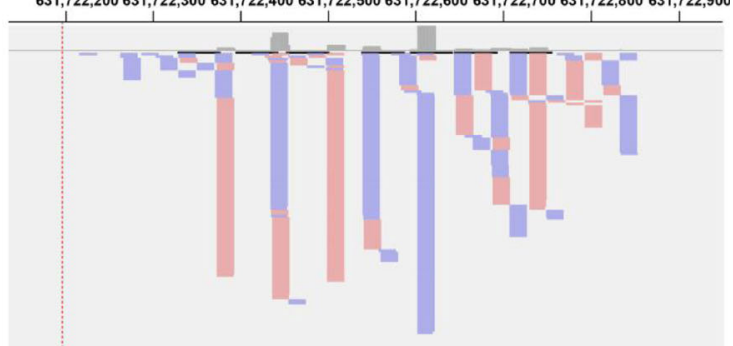

(D) Target: 5, UgaaduaUuggaggaAaAcaAa 3,
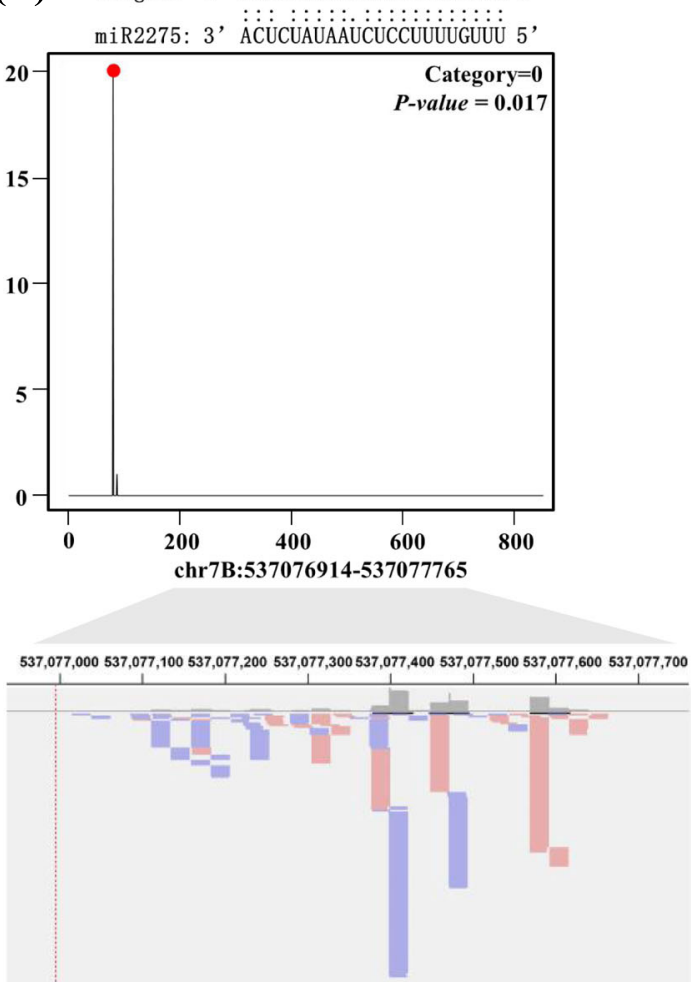

Fig. 7 Target plots (T-plots) of miRNAs characterized in the degradome datasets. The abundance of signature tags was plotted along the indicated transcripts. The red dots indicate the predicted cleavage sites on the $x$-axis, and the black lines indicate the signatures produced by miRNA-directed cleavage. In the bottom panel, the red or blue lines represents the positive or negative strand orientation of the phasiRNAs 
SRR3680677) and 9.65\% (353 out of 3659 in SRR3680678). For miR2275, few 24-PHAS were detected to be cleaved in the control and cold stressed samples. Only 0.18\% (two out of 1122 in SRR3690680) and 0.38\% (four out of 1052 in SRR3680679) of the 24-PHAS in the cold stressed samples were validated to be cleaved by miR2275, while in the control samples, there were slightly more target sites of 24-PHAS than in the cold stress samples, as confirmed by cleavage of miR2275, i.e. 3.16\% (45 out of 1421 in SRR3680677) and 9.65\% (38 out of 1203 in SRR3680678). This finding provided evidences that miR2118 and miR2275 could mediate the cleavage of PHAS transcripts in wheat. The cleavage information of the degradome for the PHAS loci were listed in Supplementary Table 8, 9, 10.

\section{The expression level of PHAS loci}

The generation of phasiRNAs may depend on the expression of their precursors. To detect the expression of the PHAS precursors, we downloaded the RNA-seq datasets from the GEO database, including the reproductive tissues at DR, FM, AM and TS. The PHAS precursors were mostly expressed in the AM and TS stages, and only a few of them were expressed in the DR and FM stages (Fig. 8a).
It was coordinated with the expression of phasiRNAs. Furthermore, most of their expression levels were very low. Only 434 and 289 of PHAS with the expression level were more than one RPKM in the AM and TS stages of young spikes, respectively, and 175 of them were overlapped between the two stages (Fig. 8c). This finding indicated the specific expression of PHAS loci in different developmental stages of young spikes.

PHAS transcripts were expressed specifically in the reproductive tissues, but whether they responded to abiotic stress was still unknown until now. Taking cold stress as an example, using the transcriptomes with polyA in the DR and AM stages of young spike samples including 0 (control), 6, 12, 24 and $48 \mathrm{~h}$ (hours) after cold stress at $0{ }^{\circ} \mathrm{C}$, we identified the expression of these PHAS loci. In the cold stressed samples, most of the PHAS loci were also mainly expressed in the AM stage, and the expression level also showed a difference (Fig. $8 \mathrm{~b})$. Using the DEseq program [31] with the fold change (stressed vs control) more than two times and adjusting the P-value less than 0.01, 102, 104, 133 and 144 of PHAS were characterized as differentially expressed PHAS transcripts at $6,12,24$ and $48 \mathrm{~h}$ after cold stress at $0{ }^{\circ} \mathrm{C}$, respectively. For the differentially expressed
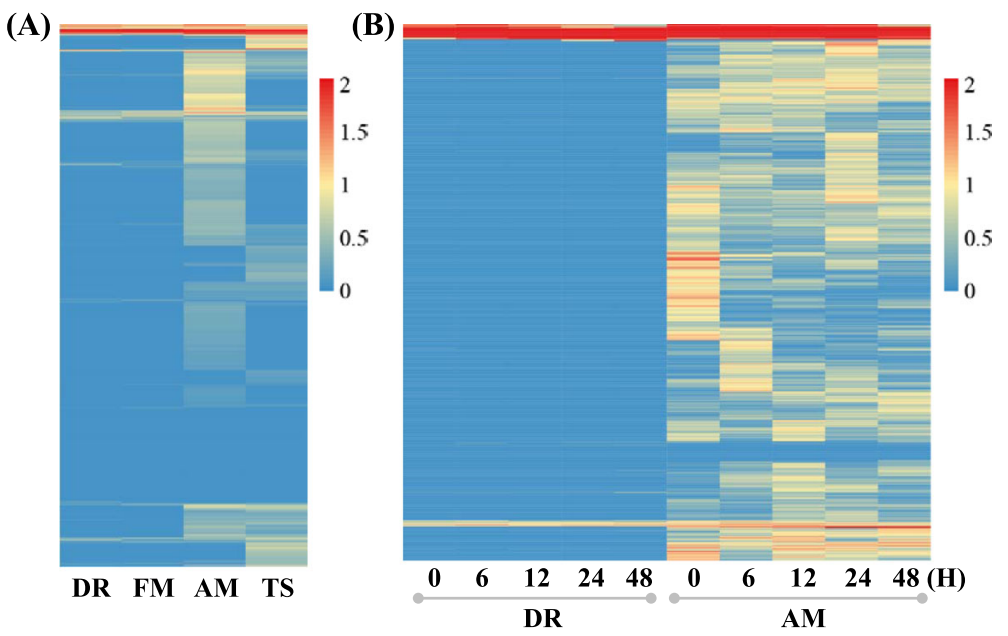

(C)
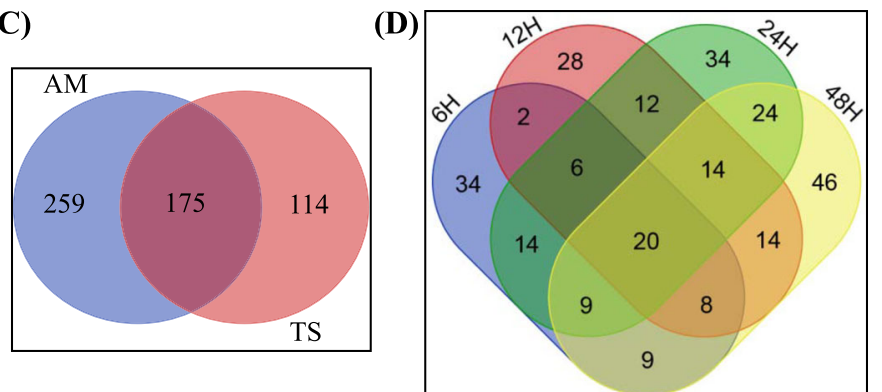

Fig. 8 Expression heatmap of PHAS transcripts in the young spike of DR, FM, AM and TS, and in the cold-stressed young spike transcriptome with polyA after $0,6,12,24$ and $48 \mathrm{~h}$ in DR and AM 
PHAS, a total of $66.67 \%$ (68 out of 102), $73.08 \%$ (76 out of 104 ), $74.43 \%$ (99 out of 133 ) and $68.06 \%$ (98 out of 144) were common at the four stressed time points (Fig. 8d). These results indicated that the PHAS transcripts responded to abiotic stress, such as cold stress.

\section{Discussion}

High conservation of the biogenesis mechanism and low conservation of PHAS sequences in plants

PhasiRNAs are present in many grass family plants including rice [3, 9, 16, 17], maize [8], Brachypodium [32], sorghum [1], foxtail millet [1], and litchi [15]. They play important roles during anther development [8]. Recent studies showed that the biogenesis mechanisms of phasiRNAs and their functional modes on target genes are conserved among grasses plants [8, 9, 15-17]. Despite this, the PHAS transcripts have very high diversity in the sequence types in different grass species. By comparing these PHAS loci in wheat to the genomes of their closely related species, including Brachypodium, rice, sorghum and maize, only 17, 7, 7 and 5 PHAS sequences in wheat could be matched with the $50 \%$ identity and $50 \%$ matched length, respectively. This result indicated that PHAS is less conserved across grass species. PMST1 producing 21-PHAS triggered by miR2118, as a typical example, regulates photoperiod-sensitive male sterility in rice [23], whereas PMST1 exists only in rice without a homolog in other grasses. Thus, PHAS and phasiRNAs are highly species-specific in grasses.

There is a special class of PHAS, i.e. TAS genes. Until now, ten $T A S$ genes were characterized in plants. For TAS genes, only some of them have high conservation, and most of them are species-specific and involved in reproductive process and biotic or abiotic stresses [2, 33, 34]. TAS1 and TAS2, which are initiated by miR173, are conserved in eudicots $[33,34]$. TAS3, which is triggered by miR390, is conserved in land plants [35]. Some of the TAS genes are family-specific. TAS5, TAS9 and TAS10 are Solanaceae-specific [36, 37]. The other TAS genes are species-specific. The TAS4 gene only exists in Arabidopsis [38]. TAS6 only exists in moss [39], and TAS7-10 only exists in grapevine [40]. These TAS genes are involved in various biological processes, such as Auxin signaling, heat and chilling response [2].

Another category of $P H A S$ is involved in regulating plant natural immunity. The regulation mechanism is also conserved both in eudicots and monocots. However, the trigger miRNAs are not conserved between eudicots and monocots. In eudicots and gymnosperm, miR472, miR482/2118, mir6024, and miR1507 can target NB-LRR transcripts and initiate the generation of phasiRNAs [41]. Most of these NB-LRR genes contain the TIR domains [42]. In monocots, very few miRNAs were identified to target the $N B-L R R$ genes [41]. Recently, in
Triticum, several miRNA families, such as miR9863, miR3117, miR3084, miR5071 and miR7757, were characterized to target $N B-L R R$ transcripts and trigger the production of phasiRNAs in wheat [41]. And most of them are absent or lost function in other grass species. In Triticum, there is also variation in the expression level or copy number for the five miRNAs [41]. The relationship between miRNAs and PHAS may frequently become absent and present during the evolution of plants.

There are no common miRNAs in eudicots or monocots that regulate the $N B-L R R$ genes. Intriguingly, miR2118, a common miRNA in eudicots and monocots diverged to neofunctionalization. In eudicots, miR2118/482 was found to mostly target $N B-L R R$ genes and initiate the generation of 21-nt phasiRNAs [13]. In contrast, miR2118 in monocots mostly targets numerous noncoding sequences and triggers the generation of 21-nt phasiRNAs [3, 8, 16]. MiR2118 in eudicots is involved in plant immunity response [13], while in monocots, miR2118 plays an important role in anther development [8, 9]. In addition, miR2118 in grasses such as rice [3] and wheat (Fig. 6) undergoes a special tandem repeat expansion. The function variation of miR2118 in the plant evolution process may be due to the target sequence variation. The sequences of the $N B-L R R$ genes rapidly diversified between eudicots and monocots [42]. For example, the TIR domain is present in most eudicots but absent in monocots. The domain loss or sequence variation of the $N B-L R R$ genes may lead to the functional variation of their regulator.

\section{Dynamic evolution of PHAS loci and their trigger MiRNAs in Triticum}

In grasses, numerous PHAS loci have been identified in maize [8], rice [3,9] and wheat. Most of them derived from the non-coding regions or intergenic regions (Supplementary Fig. 3). These PHAS loci have very high species specificity, as described in section 3.1. The Triticum species had a small time-scale in the divergence history. In Triticum, the progenitors of wheat AA, and DD diverged approximately four million years ago (mya). $\mathrm{AABB}$ and $\mathrm{AA}$ diverged approximately 0.5 mya, and AABBDD were nascent approximately 10,000 years ago. In the small time-scale of evolution, the PHAS loci still have high divergence. Most of the PHAS loci (76.09 86.22\%) were singletons with only one homoeolog among the three subgenomes, and others possessed triplet and duplet homoeologs in wheat (Supplementary Fig. 5). This study demonstrated the lower conservation of PHAS among the three subgenomes. Approximately $22-25 \%, 61-62 \%$ and $37-44 \%$ of PHAS in AABBDD could be mapped to the AA, AABB and DD genomes, respectively. The lower identity of $P H A S$ loci among subA, subB and subD indicated high heterogenization among the three subgenomes. Intriguingly, most of the 
PHAS mapped to AA, AABB and DD were located in the subA, subA \& $B$ and subD genomes of wheat, respectively. This indicated that the variation of $P H A S$ among subA, subB and subD may be after the divergence of the $\mathrm{AA}, \mathrm{BB}$ and $\mathrm{DD}$ species and may occur before the synthesized AABB and AABBDD (the left panel of Fig. 9). The subgenome plasticity may contribute less to the divergence of PHAS among the three subgenomes (the left panel of Fig. 9). The PHAS that were not mapped to the AA, AABB or DD genomes may be the nascent $P H A S$ after the yield of wheat. Overall, the noncoding PHAS loci may have a much higher evolution rate than the protein-coding genes.

The trigger miRNAs of PHAS were also expanded following genome expansion or polyploidization. The members of miR2118 and miR2275 in diploid, tetraploid and hexaploid were progressively increased and positively related to the ploidy. This result indicated the lower subgenome plasticity of the miRNA trigger among the three sets of genomes in wheat. More miR2118 was distributed in most subB chromosomes in AABB and
AABBDD than in subA and subD (Fig. 6a and Supplementary Fig. 7). However, there was no evidence to deduce the occurrence times of miR2118 expansion in the subB genome. Nevertheless, in chr5B of the AABBDD genome, there were 35 members of miR2118 (Fig. 6a), which was more than the 17 members of miR2118 in chr5B of the AABB genome (Supplementary Fig. 7). This indicated the special expansion of miR2118 in chr5B of AABBDD genomes, and the expansion may occur after the synthesis of hexaploid wheat (right panel of Fig. 9).

The expansion of miR2118 in Triticum and the other grass species may be associated with the numerous PHAS. Increasing the number of copies of miR2118 may enhance the ability to regulate PHAS and yield phasiRNAs (the middle panel of Fig. 9). After initiating the cleavage of the mRNA precursors of phasiRNAs, the secondary 21-nt phasiRNAs can function in cis to target their own precursors, which was observed in both rice and maize [9]. The simultaneously expanding and subgenome plasticity in both miRNAs and PHAS of the polyploid Triticum species may indicate their co-evolution.

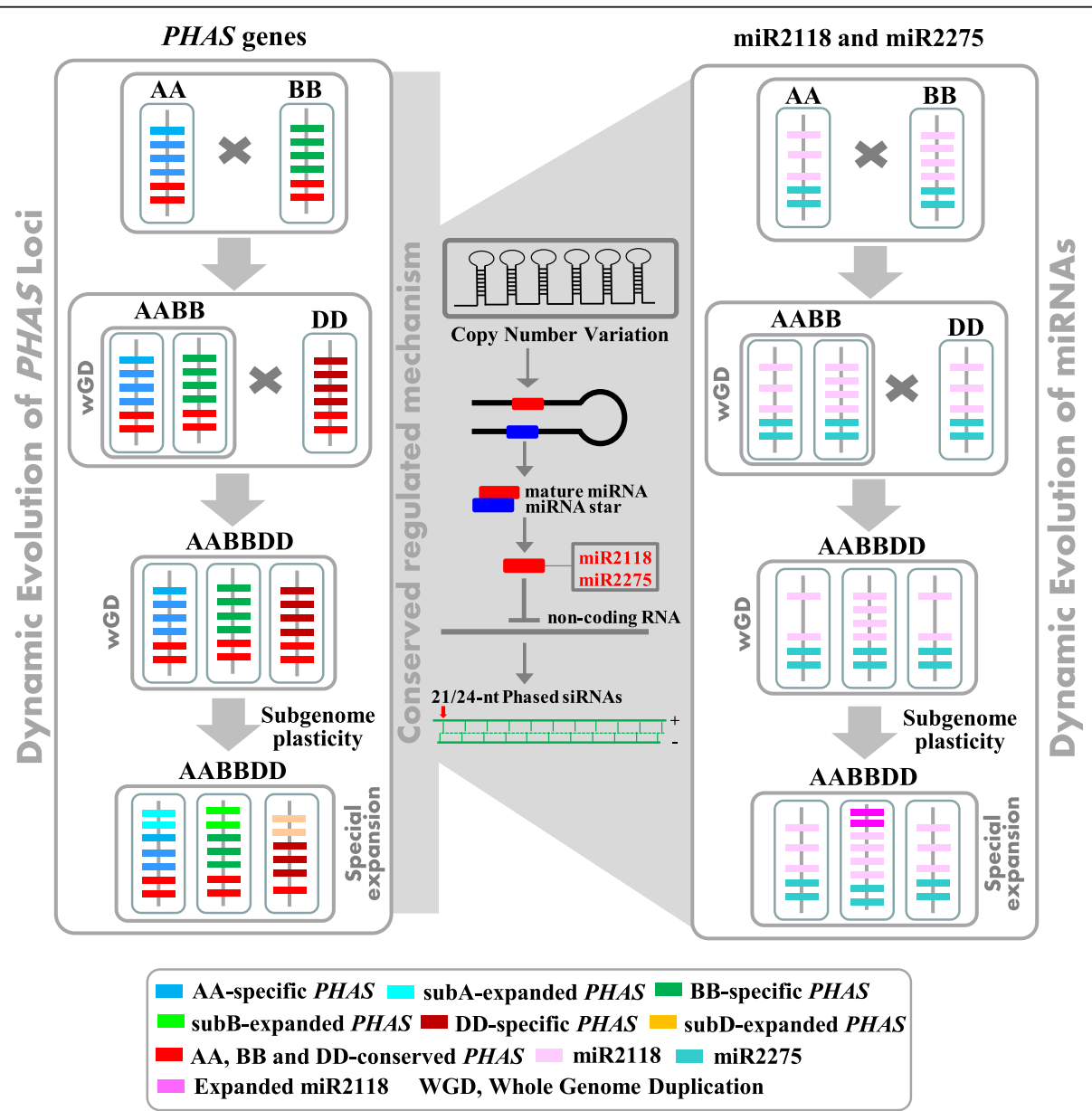

Fig. 9 Dynamically evolved PHAS (the left panel) and their regulators miR2118 and miR2275 (the right panel) in the diploid, tetraploid and hexaploid Triticum species 


\section{PHAS loci involved in male sterility}

Numerous PHAS loci were observed in male reproductive organs, such as young spikes and anthers in grasses. The PHAS loci were closely related to the fertility of the anther. Direct evidence is that the presence of PMST1 showed that PHAS was involved in male sterility in rice [23]. Indirect evidence is the mutants of genes in the biogenesis pathway of phasiRNAs. The DCL4 mutants in rice resulted in severe developmental defects in the spikelet, such as slight opening between the lemma and the palea. The lemma was partially or completely degenerated to the awn, and the strong loss-of-function transgenic plants were sterile [43]. A marked reduction of 21nt phasiRNAs was observed in the osdcl4-1 panicles [16]. The OsDCL3b RNAi lines led to reduced pollen fertility, seed setting rate and decreased grain yield in rice [44]. In addition, osdcl3b mutants specifically affected the generation of 24-nt phasiRNAs [16]. The osrdr6-1 mutant was temperature sensitive and exhibited spikelet defects. The osrdr6-1 mutants had a strong impact on the accumulation of both 21- and 24-nt phasiRNAs [17]. MEIOSIS ARRESTED AT LEPTOTENE1 (MEL1), a rice AGO, was shown to function in the development of pre-meiotic and meiotic procession, which preferred to load 24-nt phasiRNAs that bear a $5^{\prime}$-terminal cytosine [18]. In addition, mel1 mutants also behaved sterile phenotype [45]. In the micorspore mother cell (MMC) stage and meiotic prophase (MP) stage of male sterile wheat, the miR2275-3p was the significantly down-regulated compared to the fertility lines and the 24-nt siRNAs also had lower abundance in male sterile wheat lines [46]. In our study, the 24-nt phasiRNAs and miR2275 were abundant simultaneously in the TS stage (Fig. 1c-d), and validated by the degradome datasets to recognize the target sites (Fig. 7c-d \& Supplementary Tables 9, 10,11) and mediate the generation of phasiRNAs. Therefore, miR2275 and 24-nt phasiRNAs may be also associated with the male sterility in wheat. The multiple copy number of miR2275 in the genome of wheat (Fig. 6a) also indicated the importance of phasiRNAs in the evolution of plants. However, how PHAS leads to male sterility remains unclear. Whether all of the PHAS loci or phasiRNAs are associated with anther development or whether only several key PHAS loci or phasiRNAs play important roles is also unclear. The resolution of mysteries of PHAS in anther development remains a challenge for future studies.

PHAS or phasiRNAs may be responsive to abiotic stresses The PHAS loci were preferentially identified in reproductive organs in grass species, which indicated their expression preference. Similar to phasiRNAs, few PHAS transcripts were expressed in the DR and FM of young spikes (Fig. 8a \& b), which were also found in maize [8] and rice [9]. Most of the PHAS in reproductive organs in wheat had low expression levels, and only 10.49 and $4.27 \%$ of the PHAS in the AM and TS stages had expression levels with RPKM $>=1$ (Fig. 8c). The expression level between AM and TS was also different (Fig. 8c). In addition, 274 PHAS in AM were differentially expressed at $6,12,24$, and $48 \mathrm{~h}$ after cold stress at $0{ }^{\circ} \mathrm{C}$ (Fig. 8d). This result indicated that PHAS not only involved in anther development but also could respond to cold stress. In addition to cold stress, the PHAS may also be sensitive to high temperatures in the development of anthers or flowers. The phenotype of osrdr6 mutants was temperature dependent in rice. The phenotypic severity of osrdr6-1 was enhanced with increased growth temperature under the fixed photoperiod. At lower temperatures of $28^{\circ} \mathrm{C}$ or $30^{\circ} \mathrm{C}$, no obvious developmental defect or only $10 \%$ of the mutant spikelets showing a slight opening between lemma and palea was observed. At higher temperatures, the phenotypes of the mutant spikelets were very severe. At $32^{\circ} \mathrm{C}$, the lemma was lost or became a completely or partially radial, awn-like structure or both the lemma and the pelea degenerated into awn-like structures. At $34^{\circ} \mathrm{C}$, most of the lemma and pelea were defective, with shriveled or filament-like anthers. Both 21- and 24-nt phasiRNAs decreased in osrdr6 at higher temperatures [17]. Thus, phasiRNAs or PHAS transcripts may be temperature dependent or thermo-sensitive. In addition to the thermo-response, some PHAS were photoperiod-sensitive, which affected the transition of male fertility and sterility under different photoperiod conditions. $337 \mathrm{~S}$ is a male sterile wheat line that is sensitive to both long day-length/high temperature and short day-length/low temperature condition. In 337S male sterile line, the miR2275 was downregulated in MMC and MP stage [46]. MiR2275 could mediate the generation of 24-nt phasiRNAs. Thus, temperature and photoperiod conditions may affect the generation of phasiRNAs in wheat. Under long-day conditions, PMS1T was targeted by miR2118 to produce 21 nt phasiRNAs, which affected rice fertility. Under shortday conditions or under long-day conditions with the mutated target site of miR2118, PMS1T could not produce 21-nt phasiRNAs because the transcript of PMS1T could not be recognized by miR2118 [47]. Overall, according to these features of PHAS and their associated key genes, the photothermo-sensitive genic male sterile lines may be a good model to study the further function of phasiRNAs or PHAS, and the detailed molecular mechanism of male sterility will be uncovered.

\section{Methods}

Identification of PhasiRNAs and PHAS loci

The small RNA datasets used in this study were downloaded from the NCBI GEO database (Supplementary 
Table 1). The reference genomes of AA (Triticum urartu (v1), $2 \mathrm{n}=2 \mathrm{x}=14$ ), DD (Aegilops tauschii $(\mathrm{v} 1), 2 \mathrm{n}=2 \mathrm{x}=$ 14), AABB (Triticum turgidum (v1), $2 \mathrm{n}=4 \mathrm{x}=28$ ) and AABBDD (Triticum aestivum (refv1.0), $2 \mathrm{n}=6 \mathrm{x}=42$ ) were obtained from the URGI website (https://wheaturgi.versailles.inra.fr/Seq-Repository/Assemblies). Next, the Trimmomatic program (version 0.38) was used to screen the raw small RNA datasets and remove the adaptor sequences and contaminated reads. Subsequently, the ShortStack program (version 3.8.5) [19] was used to align the cleaned data to the wheat reference genome (AABBDD). Then, the distribution of small RNAs on the reference genome was analyzed by the perl scripts, and PHAS loci were identified with phased scores of greater than $15,20,25$ or 30 . According to the genome location of PHAS, the PHAS loci were annotated. A detailed flowchart was shown in Supplementary Fig. 1.

\section{The genome distribution of PHAS loci and their trigger MiR2118 and MiR2275}

To show the distribution of PHAS loci on the wheat genome, the total number of PHAS loci in each $500 \mathrm{~kb}$ window sliding of each chromosome was calculated. Then, we used the Circos program (version 0.69-6) [48] to show the number of 21-PHAS (red lines in Figs. 2, 3 and 5) and 24-PHAS (blue lines in Figs. 2, 3 and 5) loci in each chromosome in the wheat genome. The homoeologous chromosomes were filled with the same colors as the rainbow chromosomes (Figs. 2, 3 and 5). The black lines in the rainbow chromosomes indicated the PHAS loci. The homoeologous PHAS loci among the homoeologous chromosomes were linked with the same color lines, and others were linked with different color lines (Fig. 3).

To identify miR2118 and miR2275, the mature sequences of the two families of miRNAs were downloaded from the miRBase website (http://www.mirbase.org/). Then, we mapped these sequences to the AA, DD, $\mathrm{AABB}$, and AABBDD genomes using the BLAST program [49] with perfect matches. The distributions of miR2118 and miR2275 on each chromosome were drawn with the Mapchart program of the $\mathrm{R}$ package [50].

\section{Target analysis of PHAS transcripts}

To understand whether the PHAS loci were regulated by miR2118 and miR2275 in wheat, we used the Targetfinder program [28] to predict the miRNA target sites in the PHAS sequences with scores less than four. To validate whether the miRNAs can indeed cleave the predicted targets, we downloaded the degradome (SRP076763) datasets from the GEO database, including the control and cold treatment datasets [29]. The degradome datasets with the control and cold stress samples corresponded to the small RNA datasets for the control (SRR3680677 and SRR3680678) and cold stress samples (SRR3680679 and SRR3690680). Then, we used the two degradome libraries to confirm the target cleavage sites in the identified PHAS sequences in their corresponding control and cold stressed small RNA datasets with the CleaveLand program [30]. The cleavage sites were classified into $0,1,2,3$, and 4 categories according to the abundance of reads in the cleaved sites along the whole transcripts with a P-value less than 0.05 . At the cleaved sites, the categories represented the following categories: category 4 , only one read located at that position; category $3,>1$ read but below or equal to the average depth of coverage on the transcript; category $2,>1$ read above the average depth but not the maximum on the transcript; category $1,>1$ read, equal to the maximum on the transcript when there was $>1$ position at the maximum value; and category $0,>1$ read equal to the maximum on the transcript when there was just 1 position at the maximum value. Here, only these cleavages with the category $<=2$ and $P$-value $<=0.05$ were selected for further study.

\section{The expression level of PHAS transcripts}

To understand the expression level of PHAS transcripts, we downloaded the transcriptome data of DR (SRR5464507 and SRR5464508), FM (SRR5464515 and SRR5464518), AM (SRR5464519 and SRR5464520) and TS (SRR5464523 and SRR5464524) stages of young spikes, which corresponded to the following small RNA datasets: DR (SRR5460930 and SRR5460939), FM (SRR5460941 and SRR5460949), AM (SRR5460967 and SRR5460972) and TS (SRR5461176 and SRR5461177), respectively. Then, the RSEM program [51] was used to calculate the expression value (RPKM) of the PHAS transcripts. To investigate whether these PHAS loci responded to abiotic stress, we compared the transcriptomes of control and cold stressed samples in the DR and AM of young spikes. Then, we used the DEseq program [31] to calculate the differentially expressed transcripts. The heatmaps of the PHAS transcripts were drawn using the Pheatmap program in the $\mathrm{R}$ package.

\section{Conclusions}

In summary, our results provided the first PHAS profiles in the young spike of wheat. They were also triggered by miR2118 and miR2275 to generate the phasiRNAs, which provided another evidence for the conservation of PHAS loci in generation and regulation mechanism of grasses. The increase of genome ploidy was the major drive force for the expansion of PHAS loci and their trigger miRNAs in Triticum. The sequence variations and biased distribution of PHAS in these genomes of AA, 
$\mathrm{DD}, \mathrm{AABB}$ and $\mathrm{AABBDD}$ suggested their origination and diversity with rapid evolution in a small time-scale. Further study of the molecular mechanism for these PHAS loci may improve our understanding of the function of phasiRNAs in the male reproductive developments of modern polyploid wheat.

\section{Supplementary information}

Supplementary information accompanies this paper at https://doi.org/10. 1186/s12864-020-6582-4

Additional file 1: Supplementary Figure 1. The flowchart of the identification of PHAS loci.

Additional file 2: Supplementary Figure 2. The number of PHAS loci in different tissues, including vegetative tissues such as leaves and reproductive tissues such as grain, spikelet, seeds, young spikes and anthers.

Additional file 3: Supplementary Figure 3. The proportions of 21 and 24-PHAS located in gene regions, repeat sequence regions and intergenic regions.

Additional file 4: Supplementary Figure 4. Venn diagram of 21- (A) and 24-PHAS loci (B) in AM, TS, FHM, MIT and MP

Additional file 5: Supplementary Figure 5. Distribution of PHAS copies in the AM, TS, FHM, MIT and MP stages of hexaploid wheat.

Additional file 6: Supplementary Figure 6. The correlation between the percentage of mapped 21- and 24-PHAS to the AA, DD and AABB genomes and the times of ploidy.

Additional file 7: Supplementary Figure 7. The distribution of miR2118 (red lines) and miR2275 (blue lines) in each chromosome of the AABB genome.

Additional file 8: Supplementary Figure 8. The distribution of miR2118 (red lines) and miR2275 (blue lines) in the AA (A) and DD (B) genomes.

Additional file 9: Supplementary Table 1. Identified 21- and 24-PHAS with phased scores greater than 15, 20, 25 and 30 in 261 small RNA samples.

Additional file 10: Supplementary Table 2. The number and proportion of mapped 21- and 24-PHAS with scores greater than 30 for the $A A, A A B B$ and DD genomes.

Additional file 11: Supplementary Table 3. The predicted targets in AM with scores less than four, as determined by the Targetfinder program.

Additional file 12: Supplementary Table 4. The predicted targets in TS with scores less than four, as determined by the Targetfinder program Additional file 13: Supplementary Table 5. The predicted targets in FHM with scores less than four, as determined by the Targetfinder program.

Additional file 14: Supplementary Table 6. The predicted targets in MIT with scores less than four, as determined by the Targetfinder program.

Additional file 15: Supplementary Table 7. The predicted targets in MP with scores less than four, as determined by the Targetfinder program.

Additional file 16: Supplementary Table 8. The target cleavage information in AM (control: SRR3690677) from the degradome datasets.

Additional file 17: Supplementary Table 9. The target cleavage information in AM (control: SRR3690678) from the degradome datasets.

Additional file 18: Supplementary Table 10. The target cleavage information in AM (cold stress: SRR3690679) from the degradome datasets.

Additional file 19: Supplementary Table 11. The target cleavage information in AM (cold stress: SRR3690680) from the degradome datasets.

\section{Abbreviations}

AM: Anther primordia stage; DR: Double-ridge stage; FHM: Free haploid microspores stage; FM: The stage of appearance of the floret meristems; MIT: Mitosis stage; MP: Mature pollen; NT: Nucleotide; PHAS: PhasiRNA precursors; PhasiRNAs: Phased, secondary small interfering RNAs; TPM: Transcripts Per Million; TS: Tetrads stage

Acknowledgments

Not applicable.

\section{Authors' contributions}

Conceptualization, R.Z. and S.Z.; methodology, S.H., S.L., and R.Z.; software, S.H. and S.L.; validation, R.Z., S.H., G.S. and Y.L.; formal analysis, G.S., Y.L., J.L.; investigation, J.G, T.G and D.L.; resources, W.L., J.L.; data curation, R.Z. S.H., and S.L., and S.Z.; writing the original draft preparation, R.Z.; writing review and editing, R.Z., S.L., S.H., S.Z., and G.L.; visualization, S.L. and S.H.; supervision, R.Z., G.L. and S.Z.; project administration, G.L.; funding acquisition, R.Z., S.Z., G.L., and G.S. All authors have read and approved the manuscript, and ensure that this is the case.

\section{Funding}

This research was funded by the Ministry of Agriculture of China (2018ZX08009-10B), the National Natural Science Foundation of China (31501312, 31601301 and 31701428) in the design of the study, the collection of data and writing the manuscript. The obtained of the sequencing data was supported by the Science and Technology Innovation Project of Shandong Academy of Agricultural Sciences (CXGC2016C09 and CXGC2019G02), and Agricultural Variety Improvement Project of Shandong (2019LZGC015). The data analysis and interpretation were supported by the Key R\&D Programme of Shandong Province (2017GNC10113), and the Ministry of Science and Technology of China (2016YFD0100500).

Availability of data and materials

These small RNA, transcriptome, and degradome datasets were downloaded from the NCBI GEO and SRA database. The datasets supporting the conclusions of this article are included within the article and its additional files.

Ethics approval and consent to participate

Not applicable.

\section{Consent for publication}

Not applicable.

\section{Competing interests}

The authors declare that they have no competing interests.

\section{Author details}

${ }^{1}$ Crop Research Institute, Shandong Academy of Agricultural Sciences, Jinan 250100, Shandong, China. ${ }^{2}$ Key Laboratory of Wheat Biology and Genetic Improvement on North Yellow and Huai River Valley, Ministry of Agriculture, Jinan 250100, Shandong, China. ${ }^{3}$ National Engineering Laboratory for Wheat and Maize, Jinan 250100, Shandong, China. ${ }^{4} \mathrm{BG}$ Institute of Applied Agriculture, BGI-Shenzhen, Shenzhen 518120, China.

Received: 17 October 2019 Accepted: 17 February 2020

Published online: 04 March 2020

References

1. Fei $\mathrm{Q}$, Xia R, Meyers BC. Phased, secondary, small interfering RNAs in posttranscriptional regulatory networks. Plant Cell. 2013;25(7):2400-15.

2. Deng P, Muhammad S, Cao M, Wu L. Biogenesis and regulatory hierarchy of phased small interfering RNAs in plants. Plant Biotechnol J. 2018;16(5):965-75

3. Johnson C, Kasprzewska A, Tennessen K, Fernandes J, Nan GL, Walbot V, Sundaresan V, Vance V, Bowman LH. Clusters and superclusters of phased small RNAs in the developing inflorescence of rice. Genome Res. 2009; 19(8):1429-40.

4. Grivna ST, Beyret $E$, Wang Z, Lin H. A novel class of small RNAs in mouse spermatogenic cells. Genes Dev. 2006;20(13):1709-14. 
5. Li F, Pignatta D, Bendix C, Brunkard JO, Cohn MM, Tung J, Sun H, Kumar P, Baker B. MicroRNA regulation of plant innate immune receptors. Proc Nat Acad Sci U S A. 2012;109(5):1790-5.

6. Liu J, Cheng X, Liu D, Xu W, Wise R, Shen QH. The miR9863 family regulates distinct Mla alleles in barley to attenuate NLR receptor-triggered disease resistance and cell-death signaling. PLoS Genet. 2014;10(12):e1004755.

7. Guo G, Liu X, Sun F, Cao J, Huo N, Wuda B, Xin M, Hu Z, Du J, Xia R, et al. Wheat miR9678 affects seed germination by generating phased siRNAs and modulating Abscisic acid/gibberellin signaling. Plant Cell. 2018;30(4): 796-814.

8. Zhai J, Zhang H, Arikit S, Huang K, Nan GL, Walbot V, Meyers BC Spatiotemporally dynamic, cell-type-dependent premeiotic and meiotic phasiRNAs in maize anthers. Proc Natl Acad Sci U S A. 2015;112(10):3146-51.

9. Tamim S, Cai Z, Mathioni SM, Zhai J, Teng C, Zhang Q, Meyers BC. Cisdirected cleavage and nonstoichiometric abundances of 21-nucleotide reproductive phased small interfering RNAs in grasses. New Phytol. 2018; 220(3):865-77.

10. Kakrana A, Mathioni SM, Huang K, Hammond R, Vandivier L, Patel P, Arikit S, Shevchenko O, Harkess AE, Kingham B, et al. Plant 24-nt reproductive phasiRNAs from intramolecular duplex mRNAs in diverse monocots. Genome Res. 2018;28(9):1333-44.

11. Zhang $Y$, Xia R, Kuang $H$, Meyers BC. The diversification of plant NBS-LRR defense genes directs the evolution of MicroRNAs that target them. Mol Biol Evol. 2016;33(10):2692-705

12. Cakir O, Candar-Cakir B, Zhang B. Small RNA and degradome sequencing reveals important microRNA function in Astragalus chrysochlorus response to selenium stimuli. Plant Biotechnol J. 2016;14(2):543-56.

13. Zhai J, Jeong DH, De Paoli E, Park S, Rosen BD, Li Y, Gonzalez AJ, Yan Z, Kitto SL, Grusak MA, et al. MicroRNAs as master regulators of the plant NBLRR defense gene family via the production of phased, trans-acting siRNAs. Genes Dev. 2011;25(23):2540-53.

14. Wu XM, Kou SJ, Liu YL, Fang YN, Xu Q, Guo WW. Genomewide analysis of small RNAs in nonembryogenic and embryogenic tissues of citrus: microRNA- and siRNA-mediated transcript cleavage involved in somatic embryogenesis. Plant Biotechnol J. 2015;13(3):383-94.

15. Xia R, Chen C, Pokhrel S, Ma W, Huang K, Patel P, Wang F, Xu J, Liu Z, Li J, et al. 24-nt reproductive phasiRNAs are broadly present in angiosperms. Nat Commun. 2019;10(1):627.

16. Song X, Li P, Zhai J, Zhou M, Ma L, Liu B, Jeong DH, Nakano M, Cao S, Liu C, et al. Roles of DCL4 and DCL3b in rice phased small RNA biogenesis. Plant J. 2012;69(3):462-74.

17. Song X, Wang D, Ma L, Chen Z, Li P, Cui X, Liu C, Cao S, Chu C, Tao Y, et al. Rice RNA-dependent RNA polymerase 6 acts in small RNA biogenesis and spikelet development. Plant J. 2012;71(3):378-89.

18. Komiya R, Ohyanagi H, Niihama M, Watanabe T, Nakano M, Kurata N, Nonomura K. Rice germline-specific Argonaute MEL1 protein binds to phasiRNAs generated from more than 700 lincRNAs. Plant J. 2014;78(3):385-97.

19. Axtell MJ. ShortStack: comprehensive annotation and quantification of small RNA genes. RNA. 2013;19(6):740-51.

20. Feng N, Song G, Guan J, Chen K, Jia M, Huang D, Wu J, Zhang L, Kong X, Geng $\mathrm{S}$, et al. Transcriptome profiling of wheat inflorescence development from spikelet initiation to floral patterning identified stage-specific regulatory genes. Plant Physiol. 2017;174(3):1779-94.

21. Browne RG, lacuone S, Li SF, Dolferus R, Parish RW. Anther morphological development and stage determination in Triticum aestivum. Front Plant Sci. 2018;9:228.

22. Thomas BC, Pedersen B, Freeling M. Following tetraploidy in an Arabidopsis ancestor, genes were removed preferentially from one homeolog leaving clusters enriched in dose-sensitive genes. Genome Res. 2006:16(7):934-46.

23. Sankoff D, Zheng C, Zhu Q. The collapse of gene complement following whole genome duplication. BMC Genomics. 2010;11:313.

24. Pont C, Murat F, Confolent C, Balzergue S, Salse J. RNA-seq in grain unveils fate of neo- and paleopolyploidization events in bread wheat (Triticum aestivum L.). Genome Biol. 2011;12(12):R119.

25. International Wheat Genome Sequencing C. A chromosome-based draft sequence of the hexaploid bread wheat (Triticum aestivum) genome. Science. 2014;345(6194):1251788.

26. Pfeifer M, Kugler KG, Sandve SR, Zhan B, Rudi H, Hvidsten TR, International Wheat Genome Sequencing C, Mayer KF, Olsen OA. Genome interplay in the grain transcriptome of hexaploid bread wheat. Science. 2014; 345(6194): 1250091.
27. Kozomara A, Birgaoanu M, Griffiths-Jones S. miRBase: from microRNA sequences to function. Nucleic Acids Res. 2019;47(D1):D155-62.

28. Bo X, Wang S. TargetFinder: a software for antisense oligonucleotide target site selection based on MAST and secondary structures of target mRNA. Bioinformatics. 2005;21(8):1401-2.

29. Song G, Zhang R, Zhang S, Li Y, Gao J, Han X, Chen M, Wang J, Li W, Li G. Response of microRNAs to cold treatment in the young spikes of common wheat. BMC Genomics. 2017;18(1):212.

30. Addo-Quaye C, Miller W, Axtell MJ. CleaveLand: a pipeline for using degradome data to find cleaved small RNA targets. Bioinformatics. 2009; 25(1):130-1.

31. Love MI, Huber W, Anders S. Moderated estimation of fold change and dispersion for RNA-seq data with DESeq2. Genome Biol. 2014; 15(12):550.

32. Jeong DH, Schmidt SA, Rymarquis LA, Park S, Ganssmann M, German MA, Accerbi M, Zhai J, Fahlgren N, Fox SE, et al. Parallel analysis of RNA ends enhances global investigation of microRNAs and target RNAs of Brachypodium distachyon. Genome Biol. 2013;14(12):R145.

33. Yoshikawa M, Iki T, Numa H, Miyashita K, Meshi T, Ishikawa M. A short open Reading frame encompassing the MicroRNA173 target site plays a role in trans-acting small interfering RNA biogenesis. Plant Physiol. 2016;171(1):359-68

34. Li S, LiU J, LiU Z, Li X, WU F, He Y. HEAT-INDUCED TAS1 TARGET1 mediates Thermotolerance via HEAT STRESS TRANSCRIPTION FACTOR A1a-directed pathways in Arabidopsis. Plant Cell. 2014;26(4):1764-80.

35. Xia R, Xu J, Meyers BC. The emergence, evolution, and diversification of the miR390-TAS3-ARF pathway in land plants. Plant Cell. 2017;29(6): 1232-47.

36. Li F, Orban R, Baker B. SOMART: a web server for plant miRNA, tasiRNA and target gene analysis. Plant J. 2012;70(5):891-901.

37. Zuo J, Wang Q, Han C, Ju Z, Cao D, Zhu B, Luo Y, Gao L. SRNAome and degradome sequencing analysis reveals specific regulation of sRNA in response to chilling injury in tomato fruit. Physiol Plant. 2017; 160(2):142-54.

38. Guan X, Pang M, Nah G, Shi X, Ye W, Stelly DM, Chen ZJ. MiR828 and miR858 regulate homoeologous MYB2 gene functions in Arabidopsis trichome and cotton fibre development. Nat Commun. 2014;5:3050.

39. Arif MA, Fattash I, Ma Z, Cho SH, Beike AK, Reski R, Axtell MJ, Frank W. DICER-LIKE3 activity in Physcomitrella patens DICER-LIKE4 mutants causes severe developmental dysfunction and sterility. Mol Plant. 2012;5(6):1281-94.

40. Zhang C, Li G, Wang J, Fang J. Identification of trans-acting siRNAs and their regulatory cascades in grapevine. Bioinformatics. 2012; 28(20):2561-8.

41. Zhang R, Zhang S, Hao W, Song G, Li Y, Li W, Gao J, Zheng Y, Li G. LineageSpecific Evolved MicroRNAs Regulating NB-LRR Defense Genes in Triticeae. Int J Mol Sci. 2019; 20(13).

42. Zhang R, Murat F, Pont C, Langin T, Salse J. Paleo-evolutionary plasticity of plant disease resistance genes. BMC Genomics. 2014;15:187.

43. Liu B, Chen Z, Song X, Liu C, Cui X, Zhao X, Fang J, Xu W, Zhang H, Wang X, et al. Oryza sativa dicer-like4 reveals a key role for small interfering RNA silencing in plant development. Plant Cell. 2007;19(9):2705-18.

44. Liao PF, Ouyang JX, Zhang JJ, Yang L, Wang X, Peng XJ, Wang D, Zhu YL, Li $\mathrm{SB}$. OsDCL3b affects grain yield and quality in rice. Plant Mol Biol. 2019:99(3):193-204.

45. Nonomura K, Morohoshi A, Nakano M, Eiguchi M, Miyao A, Hirochika H, Kurata N. A germ cell specific gene of the ARGONAUTE family is essential for the progression of premeiotic mitosis and meiosis during sporogenesis in rice. Plant Cell. 2007;19(8):2583-94

46. Sun L, Sun G, Shi C, Sun D. Transcriptome analysis reveals new microRNAsmediated pathway involved in anther development in male sterile wheat. BMC Genomics. 2018;19(1):333.

47. Fan Y, Yang J, Mathioni SM, Yu J, Shen J, Yang X, Wang L, Zhang Q, Cai Z, $\mathrm{Xu} C$, et al. PMS1T, producing phased small-interfering RNAs, regulates photoperiod-sensitive male sterility in rice. Proc Natl Acad Sci U S A. 2016; 113(52):15144-9.

48. Krzywinski M, Schein J, Birol I, Connors J, Gascoyne R, Horsman D, Jones SJ, Marra MA. Circos: an information aesthetic for comparative genomics. Genome Res. 2009;19(9):1639-45.

49. Mount DW. Using the Basic Local Alignment Search Tool (BLAST). CSH Protoc. 2007; 2007:pdb top17. 
50. Voorrips RE. MapChart: software for the graphical presentation of linkage maps and QTLS. J Hered. 2002;93(1):77-8.

51. Li B, Dewey CN. RSEM: accurate transcript quantification from RNA-Seq data with or without a reference genome. BMC Bioinformatics. 2011;12:323.

\section{Publisher's Note}

Springer Nature remains neutral with regard to jurisdictional claims in published maps and institutional affiliations.

Ready to submit your research? Choose BMC and benefit from:

- fast, convenient online submission

- thorough peer review by experienced researchers in your field

- rapid publication on acceptance

- support for research data, including large and complex data types

- gold Open Access which fosters wider collaboration and increased citations

- maximum visibility for your research: over $100 \mathrm{M}$ website views per year

At $\mathrm{BMC}$, research is always in progress.

Learn more biomedcentral.com/submissions 